\title{
Quantum Metrology for Non-Markovian Processes
}

\author{
Anian Altherr ${ }^{1, *}$ and Yuxiang Yang ${ }^{1,2, \dagger}$ \\ ${ }^{1}$ Institute for Theoretical Physics, ETH Zürich, 8093 Zürich, Switzerland \\ ${ }^{2} \mathrm{QICI}$ Quantum Information and Computation Initiative, Department of Computer Science, \\ The University of Hong Kong, Pokfulam Road, Hong Kong
}

\begin{abstract}
Quantum metrology is a rapidly developing branch of quantum technologies. While various theories have been established on quantum metrology for Markovian processes, i.e., quantum channel estimation, quantum metrology for non-Markovian processes is much less explored. In this Letter, we establish a general framework of non-Markovian quantum metrology. For any parametrized nonMarkovian process on a finite-dimensional system, we derive a formula for the maximal amount of quantum Fisher information that can be extracted from it by an optimally controlled probe state. In addition, we design an algorithm that evaluates this quantum Fisher information via semidefinite programming. We apply our framework to noisy frequency estimation, where we find that the optimal performance of quantum metrology is better in the non-Markovian scenario than in the Markovian scenario and explore the possibility of efficient sensing via simple variational circuits.
\end{abstract}

Introduction. Quantum metrology holds the promise of an early application of quantum technologies that offer an advantage over classical ones. This advantage, nevertheless, is often sensitive to noise [1-4]. Advances in quantum metrology have been focusing on identifying the ultimate limit of parameter estimation in the presence of noise [2, 3, 5-7]. So far, the analysis has mostly been constrained to the Markovian setting and carried out within the well-established model of quantum channel estimation [Fig. 1 (a)], where various tools have been developed. In particular, it has recently been shown that the quantum advantage can be retrieved by using appropriate quantum control [8-11].

Non-Markovian quantum metrology [Fig. 1 (b)], on the other hand, is much less explored. Despite interesting findings in specific cases (see, e.g., Ref. [12$18]$ ), there has not been a systematic way to treat nonMarkovian quantum metrology. As a first step, in Ref. [17], one of us proposed a general framework for non-Markovian quantum metrology based on an information-theoretic structure, named the quantum comb [19-21], which has also been applied to a broad range of tasks including channel discrimination [22], quantum network optimization [23], Markovianity [24], and probabilistic quantum computation [25]. However, given an arbitrary non-Markovian metrology task, the core question of determining the ultimate precision limit, optimized over all possible means of quantum probe preparation and control, still remains open. This issue is pressing not only because non-Markovian processes are prevalent in physics, but also for the rapid development of quantum computing: With the advance of NISQ (near-term intermediate-scale quantum) devices [26], deeper quantum circuits will appear where memory effects of the environment are more significant. New techniques will soon be needed to test and benchmark com-

\footnotetext{
* aaltherr@ethz.ch

†yangyu@ethz.ch
}

plex noises generated by such effects.

In this Letter, we establish a general framework of assessing the precision of non-Markovian quantum metrology, quantified by the quantum Fisher information (QFI). The framework is built upon our preceding work [17], where non-Markovian metrology is modelled using quantum combs. Here we address the pivotal problem of evaluating the QFI of arbitrary quantum combs, deriving both a general formula and an algorithm that efficiently computes the QFI of arbitrary combs via semidefinite programming. As a working example, we apply the algorithm to the task of frequency estimation under non-Markovian noise, where we observe that the QFI can be increased by applying suitable quantum control to the system. We also show that, when the memory of the non-Markovian noise is not too deep, this control can be well approximated by a simple variational circuit, whose complexity grows only linearly in the memory depth. Our results extend quantum metrology to generic physical processes with memory, paving the way for various future research and applications.

Quantum Fisher information of quantum combs. Our goal is to estimate a single parameter $\theta$ from a non-Markovian process that carries this parameter. For instance, the task could be to measure the frequency discrepancy in a noisy atomic clock where the atoms are coupled to a persistent environment, or to estimate the strength of time-correlated noise in a deep quantum circuit.

Reference [17] introduced parametrized quantum combs for parameter estimation with non-Markovian processes. Consider an $\mathrm{N}$-step non-Markovian process that consists of $N$ consecutive quantum channels with partially accessible inputs and outputs concatenated by an inaccessible memory. Such a process is completely positive (CP) and thus, by the Choi-Jamiolkowski isomorphism, the non-Markovian process is uniquely characterized by a positive semidefinite Choi operator [27], referred to as its quantum comb [19-21]. For a process, the comb is obtained by inserting the second half of the vector $\sum_{n}|n\rangle|n\rangle$ into all input ports (e.g. odd-labeled 
(a)

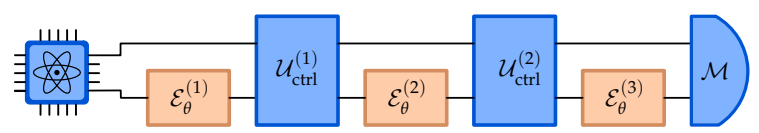

(b)

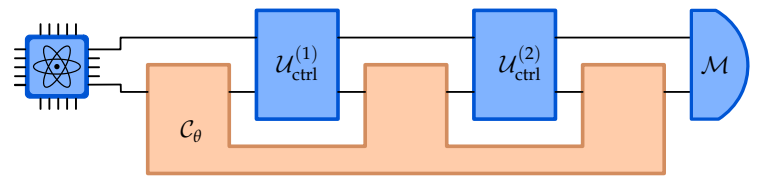

Figure 1. Markovian versus non-Markovian quantum metrology. (a): The standard, Markovian setting of quantum metrology where the goal is to estimate $\theta$ from a sequence of quantum channels (orange), and the general strategy is to prepare a probe state and apply adaptive quantum control (blue). (b): Non-Markovian quantum metrology where $\theta$ is encoded in a non-Markovian process with inaccessible memory (orange).

spaces in Fig. 2), where $\{|n\rangle\}$ is an orthonormal basis of the input ports. Conversely, an operator corresponds to the comb of a process if it satisfies a series of linear constraints (see Definition 1 below). We will therefore denote a non-Markovian process by its comb. In Fig. 2, we can see that a comb consists of a sequence of $N$ teeth (corresponding to an $N$-step process), each tooth $k=1, \ldots, N$ has an input space $\mathcal{H}_{2 k-1}$ and an output space $\mathcal{H}_{2 k}$. We denote by $\operatorname{Comb}\left[\left(\mathcal{H}_{1}, \mathcal{H}_{2}\right),\left(\mathcal{H}_{3}, \mathcal{H}_{4}\right)\right.$, $\left.\ldots,\left(\mathcal{H}_{2 N-1}, \mathcal{H}_{2 N}\right)\right]$ all combs for a given sequence of input and output ports (characterized, e.g., by odd- and even-labeled Hilbert spaces respectively). We denote by $\mathcal{L}(\mathcal{H})$ linear operators on $\mathcal{H}$ and assume all Hilbert spaces to be finite dimensional.

Definition 1 (Quantum combs [19-21]). A positive semidefinite $C \in \mathcal{L}\left(\otimes_{i=1}^{2 N} \mathcal{H}_{i}\right)$ is a comb in $\operatorname{Comb}\left[\left(\mathcal{H}_{1}, \mathcal{H}_{2}\right),\left(\mathcal{H}_{3}, \mathcal{H}_{4}\right), \ldots,\left(\mathcal{H}_{2 N-1}, \mathcal{H}_{2 N}\right)\right]$ if and only if there exist a sequence of positive semidefinite operators

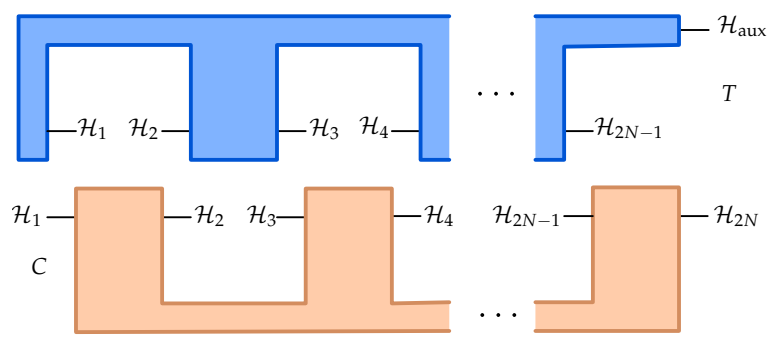

Figure 2. Quantum combs and probes. A generic quantum comb $C \in \operatorname{Comb}\left[\left(\mathcal{H}_{1}, \mathcal{H}_{2}\right), \ldots,\left(\mathcal{H}_{2 N-1}, \mathcal{H}_{2 N}\right)\right]$ (orange) consists of $N$ teeth, each representing one time step. A probe $T \in \operatorname{Comb}\left[\left(\varnothing, \mathcal{H}_{1}\right),\left(\mathcal{H}_{2}, \mathcal{H}_{3}\right), \ldots,\left(\mathcal{H}_{2 N-2}, \mathcal{H}_{2 N-1} \otimes \mathcal{H}_{\text {aux }}\right)\right]$ for $C$ (blue) is a comb that "eats" $C$ and "spits out" a quantum state on $\mathcal{H}_{2 N} \otimes \mathcal{H}_{\text {aux }}$.

$$
\begin{aligned}
& C^{(k)} \in \mathcal{L}\left(\otimes_{i=1}^{2 k} \mathcal{H}_{i}\right)(k=1, \ldots, N-1) \text { such that: } \\
& \operatorname{tr}_{2 k}\left[C^{(k)}\right]=\mathbb{1}_{2 k-1} \otimes C^{(k-1)} \quad k=2, \ldots, N-1, \\
& \operatorname{tr}_{2}\left[C^{(1)}\right]=\mathbb{1}_{1} \quad \operatorname{tr}_{2 N}[C]=\mathbb{1}_{2 N-1} \otimes C^{(N-1)} .
\end{aligned}
$$

The linear constraints in Eq. (1) are due to normalization and causality. The constraints distinguish between input and output ports (as, for example, it is always the output ports that are traced out) and ensure that the ports are causally ordered according to the labels. When $N=1$, Eq. (1) reduces to a simple normalization constraint $\operatorname{tr}_{2}[C]=\mathbb{1}_{1}$, and $\operatorname{Comb}\left[\left(\mathcal{H}_{1}, \mathcal{H}_{2}\right)\right]$ is just the collection of all quantum channels from $\mathcal{H}_{1}$ to $\mathcal{H}_{2}$.

Two combs can be interlaced with each other following the rule of the link product $[20,21]$ : Given two combs $E \in \mathcal{L}\left(\bigotimes_{m \in M} \mathcal{H}_{m}\right)$ and $F \in \mathcal{L}\left(\bigotimes_{n \in N} \mathcal{H}_{n}\right)$, the link product $E * F \in \mathcal{L}\left(\bigotimes_{s \in S} \mathcal{H}_{S}\right)$ for $S=(M \cup$ $N) \backslash(M \cap N)$, which yields a new comb, is defined by

$$
E * F:=\operatorname{tr}_{M \cap N}\left[\left(E^{T_{M \cap N}} \otimes \mathbb{1}_{N \backslash M}\right)\left(\mathbb{1}_{M \backslash N} \otimes F\right)\right],
$$

where $T_{A}$ is the partial transpose performed on Hilbert spaces with indices in $A$. An example is depicted in Fig. 3. Also note that in Fig. 2 the link product $C * T$ yields a quantum state.

For Markovian quantum metrology, the task is to find an optimal probe state so that, by inserting it to the parametrized channel, the output state has maximal QFI. Similarly, to estimate $\theta$ from a parametrized quantum comb $C_{\theta}$, the goal is to find an optimal probe $T$ consisting of state preparation and control such that $C_{\theta} * T$ is a quantum state with maximal QFI. As depicted in Fig. 2, for $C_{\theta} \in \operatorname{Comb}\left[\left(\mathcal{H}_{1}, \mathcal{H}_{2}\right), \ldots,\left(\mathcal{H}_{2 N-1}, \mathcal{H}_{2 N}\right)\right]$, the probe should be $T \in \operatorname{Comb}\left[\left(\varnothing, \mathcal{H}_{1}\right),\left(\mathcal{H}_{2}, \mathcal{H}_{3}\right)\right.$, $\left.\ldots,\left(\mathcal{H}_{2 N-2}, \mathcal{H}_{2 N-1} \otimes \mathcal{H}_{\text {aux }}\right)\right]$, with $\mathcal{H}_{\text {aux }}$ being an ancilla and $\varnothing$ denoting a trivial input space. Thus, the probe has input and output spaces complementary to the original quantum comb at the first $(N-1)$ teeth, and $C_{\theta} * T$ is a quantum state in $\mathcal{H}_{2 N} \otimes \mathcal{H}_{\text {aux }}$. It is then natural to define the QFI of a quantum comb as [17]:

$$
J\left(C_{\theta}\right)=\max _{T \in \operatorname{Comb}\left[\left(\varnothing, \mathcal{H}_{1}\right), \ldots,\left(\mathcal{H}_{2 N-2}, \mathcal{H}_{2 N-1} \otimes \mathcal{H}_{\text {aux }}\right)\right]} J\left(C_{\theta} * T\right),
$$

where, on the right-hand side, $J\left(\rho_{\theta}\right)$ is the QFI of a quantum state $\rho_{\theta}[28,29]$.

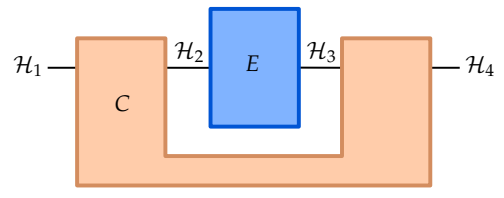

Figure 3. Link product. The link product between a comb $C \in \operatorname{Comb}\left[\left(\mathcal{H}_{1}, \mathcal{H}_{2}\right),\left(\mathcal{H}_{3}, \mathcal{H}_{4}\right)\right]$ and a quantum channel $E \in$ $\operatorname{Comb}\left[\left(\mathcal{H}_{2}, \mathcal{H}_{3}\right)\right]$ results in a channel $E * F$ from $\mathcal{H}_{1}$ to $\mathcal{H}_{4}$. 
The QFI gives a lower bound on the variance of any unbiased estimator $\hat{\theta}$ via the Cramér-Rao bound [28, 29], which can be extended to the comb setting [17]:

$$
\operatorname{Var}(\hat{\theta}) \geq \frac{1}{v J\left(C_{\theta}\right)}
$$

with $v$ being the number of times that the experiment is repeated. When $\theta$ is a single parameter, the bound is known to be achievable [28, 29].

The bound (4) establishes the comb QFI $J\left(C_{\theta}\right)$ as the pivotal quantity that determines the ultimate precision limit. Our main result consists of an analytical formula for $J\left(C_{\theta}\right)$ and an algorithm that computes it using semidefinite programming. We start by introducing the key notions:

Definition 2 (Comb conditional min-entropy [23]). For any $C \in \operatorname{Comb}\left[\left(\mathcal{H}_{1}, \mathcal{H}_{2}\right), \ldots\right.$, $\left.\left(\mathcal{H}_{2 N-1}, \mathcal{H}_{2 N}\right)\right]$, the min-entropy of the Nth tooth $\left(\mathcal{H}_{2 N-1}, \mathcal{H}_{2 N}\right)$ conditioned on the first $(N-1)$ teeth $[N-1]:=\left(\mathcal{H}_{1}, \mathcal{H}_{2}\right), \ldots,\left(\mathcal{H}_{2 N-3}, \mathcal{H}_{2 N-2}\right)$ is defined as

$$
\begin{aligned}
& H_{\min }(N \mid[N-1])_{C}:= \\
& -\log _{2} \min _{S}\left\{\lambda \in \mathbb{R} \mid \mathbb{1}_{2 N-1,2 N} \otimes \lambda S \succeq C\right\},
\end{aligned}
$$

where $S \in \operatorname{Comb}\left[\left(\mathcal{H}_{1}, \mathcal{H}_{2}\right), \ldots,\left(\mathcal{H}_{2 N-3}, \mathcal{H}_{2 N-2}\right)\right]$.

Fujiwara and Imai [30] evaluated the QFI of a quantum state $\rho_{\theta}$ via ensemble decompositions, which are vectors $\left\{\left|\phi_{\theta, i}\right\rangle\right\}$ such that $\rho_{\theta}=\sum_{i}\left|\phi_{\theta, i}\right\rangle\left\langle\phi_{\theta, i}\right|$. Note that $\left\{\left|\phi_{\theta, i}\right\rangle\right\}$ are not required to be orthonormal and thus ensemble decompositions are not unique. Then the QFI of $\rho_{\theta}$ is determined by the operators $\sum_{i}\left|\dot{\phi}_{\theta, i}\right\rangle\left\langle\dot{\phi}_{\theta, i}\right|$, where the dot stands for the partial derivative with respect to $\theta$. Here (see Ref. [31] for details) we extend the notion to combs: Since the Choi state of a quantum comb $C_{\theta}$ is Hermitian, we can find unnormalized vectors $\left|C_{\theta, i}\right\rangle$ such that

$$
C_{\theta}=\sum_{i=1}^{q}\left|C_{\theta, i}\right\rangle\left\langle C_{\theta, i}\right|
$$

where $q \geq r:=\max _{\theta} \operatorname{rank}\left(C_{\theta}\right)$. We then define the performance operator of metrology as

$$
\Omega_{\theta}(h):=4 \sum_{i=1}^{q}\left(\left|\dot{\tilde{C}}_{\theta, i}\right\rangle\left\langle\dot{\tilde{C}}_{\theta, i}\right|\right)^{T_{1 \ldots 2 N-1}} .
$$

Here $\left|\dot{\tilde{C}}_{\theta, i}\right\rangle$ is given by $\left|\dot{\tilde{C}}_{\theta, i}\right\rangle=\left|\dot{C}_{\theta, i}\right\rangle-i \sum_{j=1}^{q} h_{i j}\left|C_{\theta, j}\right\rangle$. The Hermitian matrix $h$ is of dimension $q$ for any $q \geq r$, which captures the nonuniqueness of the ensemble decomposition. One can show that (see below) it is sufficient to consider $q=r$. With these notions we have:

Theorem 1. Given a parametrized family of combs $\left\{C_{\theta}\right\}_{\theta}$ with $C_{\theta} \in \operatorname{Comb}\left[\left(\mathcal{H}_{1}, \mathcal{H}_{2}\right), \ldots,\left(\mathcal{H}_{2 N-1}, \mathcal{H}_{2 N}\right)\right]$, the QFI of the comb at $\theta$ is given as

$$
J\left(C_{\theta}\right)=d_{2 N} \min _{h \in \operatorname{Herm}\left(\mathbb{C}^{r}\right)} 2^{-H_{\min }(N \mid[N-1])_{\Omega_{\theta}(h)}}
$$

with $d_{2 N}:=\operatorname{dim}\left(\mathcal{H}_{2 N}\right)$ and Herm $\left(\mathbb{C}^{r}\right)$ being the set of $r$ dimensional Hermitian matrices.

The proof can be found in Ref. [31]. Equation (8) can be regarded as the ultimate formula of QFI, which applies to both Markovian and non-Markovian processes: By setting $C_{\theta}$ to be the tensor product of $N$ identical Choi operators, Eq. (8) yields the QFI corresponding to the optimal adaptive strategy of channel estimation (see Ref. [31] for details).

In practice, it is desirable to evaluate the comb QFI numerically. To this purpose, we develop Algorithm 1, which takes as input any parametrized family of combs and outputs the comb QFI. The detailed derivation of Algorithm 1 can be found in Ref. [31].

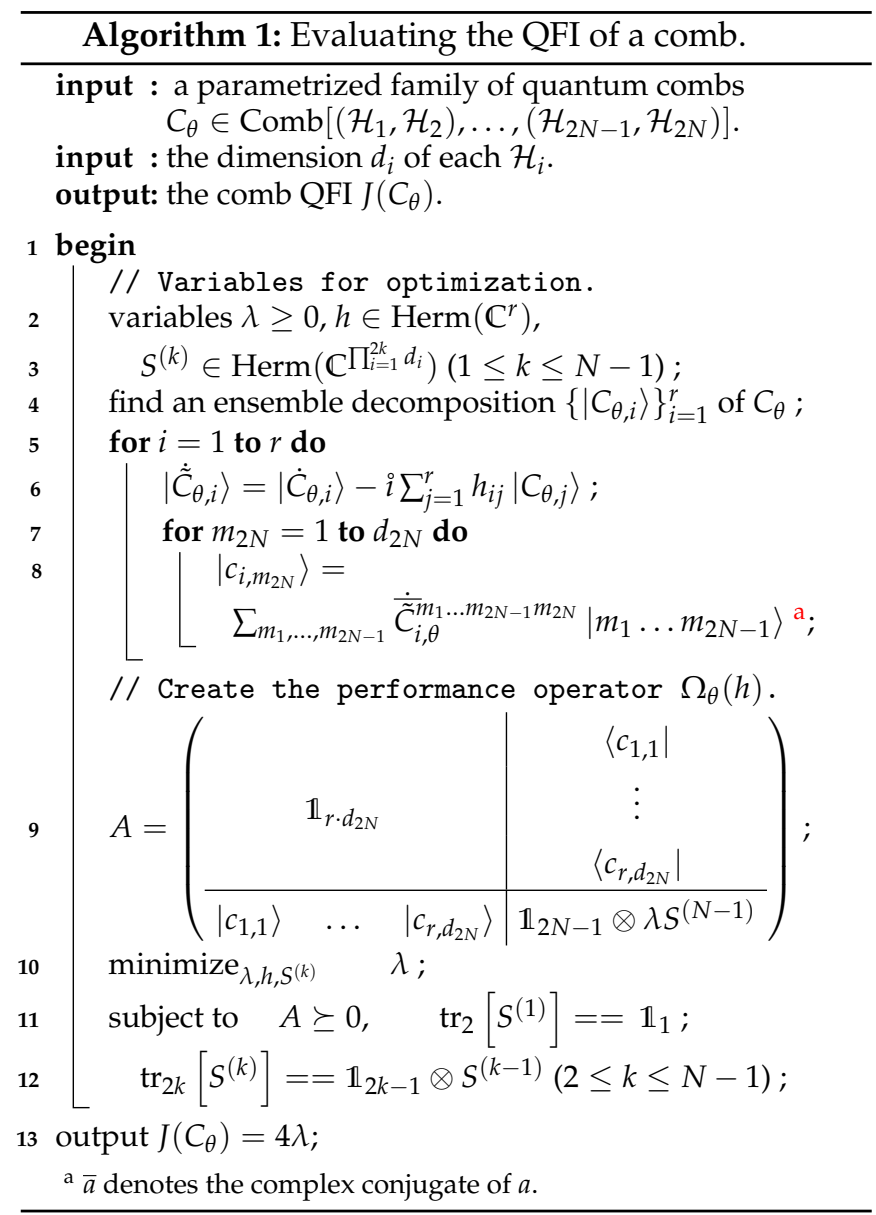

Frequency estimation under non-Markovian noise. As an example, we now apply our framework to a specific case of frequency estimation under non-Markovian noise. The task is to estimate an unknown frequency $\omega$, given $N$ sequential access to a qubit phase gate $e^{-i H t}$ with $H=\omega|1\rangle\langle 1|$. The whole process, however, is subject to non-Markovian noise, which can be modeled using a circuit model: At each of the $N$ steps, the system $\mathcal{H}_{S}$ collides with the same environment $\mathcal{H}_{E}$ via a unitary interaction $U_{\text {int }}(\tau)$ that lasts for time $\tau$ (see Fig. 4 ). The in- 


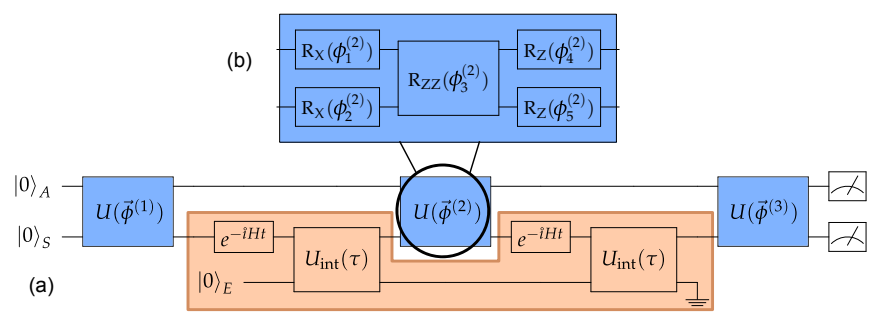

Figure 4. Noisy non-Markovian frequency estimation using a variational probe. As shown in (a), the task is to estimate $\omega$ given $N$ accesses ( $N=2$ in the figure), each of duration $t$, to a qubit system $S$ with $H=\omega|1\rangle\langle 1|$. The system is coupled to a qubit environment $E$ by an interaction $U_{\text {int }}(\tau)$, where the interaction time $\tau$ depends on $t$. To estimate $\omega$ we construct a variational probe which consists of two-qubit control operations $U\left(\vec{\phi}^{(i)}\right)$ for $i=1,2, \ldots, N+1$ and projective measurements in the computational basis. In (b) we consider a specific variational probe: The rotations are $R_{\sigma}(\phi)=e^{-i \frac{\phi}{2} \sigma}$ for $\sigma=X, Z$ and $R_{Z Z}(\phi)=e^{-i \frac{\phi}{2} Z \otimes Z}$.

teraction steers information from the system into the environment, potentially reducing the accuracy of estimation [45]. Meanwhile, the accuracy is also influenced by memory effects, as information can also flow back from the environment to the system. Such a task is beyond the existing framework of quantum metrology: One may be tempted to work out the maximal achievable QFI via a brute-force approach, by optimizing over all possible input states and controls. However, even for small systems the brute-force approach may still be unrealistic. For example, adding an ancillary system makes the QFI higher in general, but due to the underlying memory of the environment the size of the ancillary system needed to achieve optimality is hard to determine. Theorem 1 , on the other hand, involves optimization on a predetermined system and its efficiency is totally predictable.

Let us now study one concrete case. We set both the system and the environment to be single qubit. For the interaction, we choose $U_{\text {int }}(\tau)=e^{-i g \tau \text { SWAP }}$ with interaction strength $g$ and $\operatorname{SWAP}\left(|v\rangle_{S} \otimes|w\rangle_{E}\right)=|w\rangle_{S} \otimes|v\rangle_{E}$ being the swap gate. We apply Algorithm 1 to evaluate the comb QFI. For each round of the evaluation, we fix $N$ and a total time $t_{\text {tot }}$ and set $g=1, \omega=\pi / 10$, $t=\tau=t_{\text {tot }} / N$. The environment is initiated to $|0\rangle$.

Plots of the comb QFI for different $N$ and different $t_{\text {tot }}$ can be seen in Fig. 5. We compare between two strategies: The red, dashed lines stand for the QFI of the "control-free" strategy, where the probe consists of an initial state preparation and identity channels between the steps, i.e., only the input state is optimized but no intermediate control is performed between the steps. On the other hand, the QFI of the optimal strategy, where both the initial state preparation and the intermediate control are optimized, equals the comb QFI (red, solid lines) by definition. From Fig. 5, it is clear that quantum control between the steps can improve the accuracy, as there is a gap between the comb QFI (red, solid) and
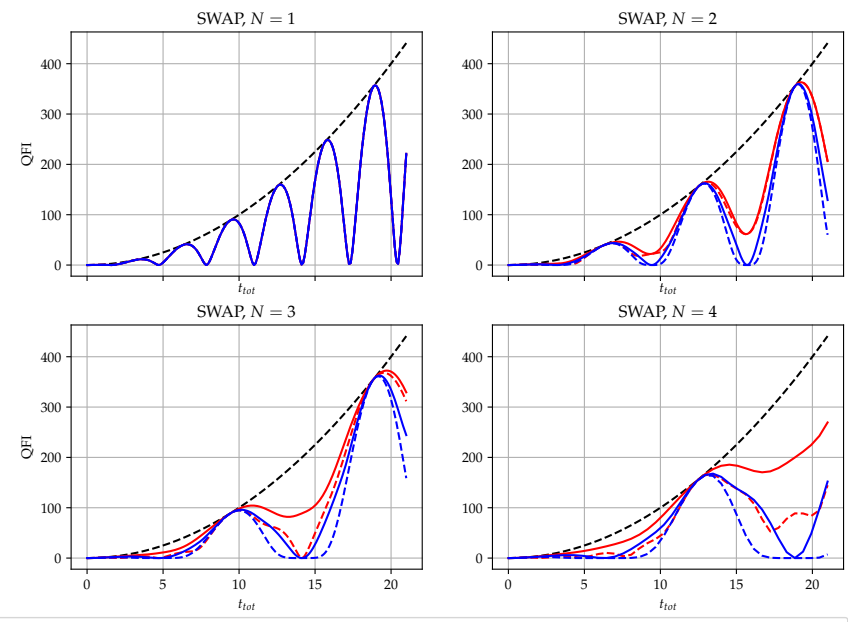

Figure 5. QFIs as a function of the total sampling time $t_{\text {tot }}$ for noisy frequency estimation. We consider the comb depicted in Fig. 4 with the SWAP interaction for different value of $N$. As a comparison, we also consider the corresponding Markovian scenario, where the environment state is traced out and reset to $|0\rangle$ after each interaction. The non-Markovian scenario with optimal control (red, solid) allows for the highest QFI. In particular, the QFI is higher than those of the scenario without control (red, dashed) and its Markovian counterpart (blue, solid). The Markovian scenario without control (blue, dashed) sees the worst performance. Notice that $N=1$ corresponds to the task of single channel estimation, where all scenarios coincide. When the interaction is trivial $\left(U_{\text {int }}(\tau)= \pm \mathbb{1}\right)$, the noiseless scaling (black, dashed) is achieved in all scenarios.

the control-free QFI (red, dashed). The gap becomes bigger when $N$ grows larger, as the non-Markovianity increases. For both strategies, the QFI of the noiseless scenario (black, dashed), which is equal to $t_{\text {tot }}^{2}$, is recovered only if the interaction $U_{\text {int }}(\tau)$ is trivial.

We can also see the difference between Markovian and non-Markovian noises: In Fig. 5, the blue, solid line corresponds to the maximal achievable QFI of the Markovian setting, where we apply the same interaction between the system and the environment but reset the environment at each step. In the Markovian setting, the whole process can be described by $N$ sequential quantum channels. One can see that the QFIs for both the optimal strategy (blue, solid) and the controlfree strategy (blue, dashed) are lower than those for the non-Markovian case, matching a recent finding in Ref. [48]. Intuitively, the reason could be that, for the non-Markovian case, the information can be retrieved from the environment using proper control. This is a phenomenon that, without doubt, deserves further investigation.

We also investigate the same task for other types of non-Markovian noises. These results can be found in Ref. [31].

High-performance metrology with variational probes. Prac- 


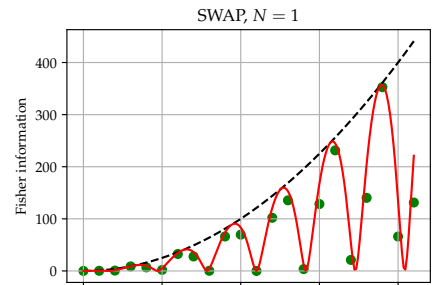

SWAP, $N=3$

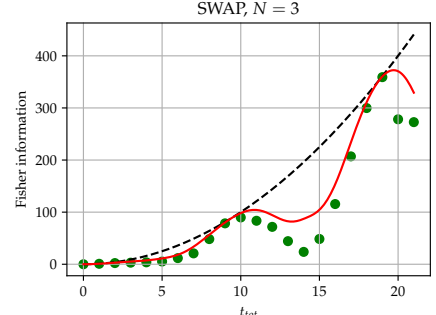

--- noiseless _ non-Markov, control

- variational

Figure 6. Performance of the variational probe. Performance of the variational probe (blue part of Fig. 4) is evaluated in terms of the Fisher information of the output probability distribution. Here the green dots are the Fisher information of the variational probe's output and the red, solid lines are the maximal attainable Fisher information.

tically, it is meaningful to consider whether the maximal QFI can be achieved by a relatively simple probe. Variational circuits, which promise a range of near-term applications thanks to its relatively simple structure, are ideal candidates. Consider the same estimation problem as the previous section. As shown in Fig. 4 b), we construct a variational probe, which consists of a fixed arrangement (i.e. ansatz) of unitary gates and measurements in the computational basis. Each unitary gate is controlled by a few variables, and the optimal probe is obtained by gradually adjusting these variables to maximise the Fisher information of the output probability distribution.

Concretely, we use a checkerboard ansatz [49] with $5(N+1)$ parameters in total. From the numerical simulation [Fig. 6], one can see that the performance of the variational probe is close to optimal, implying that it is enough to use it when $N$ is not large. The gap to the comb QFI increases with $N$, suggesting that it may require more complex circuits to approach optimality for estimating processes with stronger non-Markovianity.

Conclusion. We established a fundamental framework of non-Markovian quantum metrology. To this end, we presented a general formula of the QFI of a quantum comb and designed an algorithm which evaluates the exact value of the comb QFI. Our work opens up various directions for further research. First, Theorem 1 and Algorithm 1 can be readily applied to any concrete task of non-Markovian metrology. Second, it is interesting to explore the asymptotic performance of non-Markovian metrology using our framework and to capture unique performance limits such as the quantum Zeno limit [13]. Our method can also be extended to multi-parameter non-Markovian quantum metrology [50,51], where the tradeoff between the precisions of estimating different parameters [29, 52-57] plays a major role. Last but not least, we note that an $N$ comb, in general, has degrees of freedom that scales exponentially in $N$. Nevertheless, in many practical scenarios, it is reasonable to assume that the comb and its performance operator have only poly $(N)$ significant eigenvalues. It is then promising that an accurate and even more efficient approximate algorithm can be obtained from state-of-the-art methods of semidefinite programming, e.g., Ref. [58].

We thank Giulio Chiribella, Tobias Sutter, and Sisi Zhou for helpful discussions. This work is supported by the Swiss National Science Foundation via the National Center for Competence in Research "QSIT" as well as via project No. 200021_188541 and by the ETH Pauli Center for Theoretical Studies.
[1] S. F. Huelga, C. Macchiavello, T. Pellizzari, A. K. Ekert, M. B. Plenio, and J. I. Cirac, Improvement of frequency standards with quantum entanglement, Physical Review Letters 79, 3865 (1997).

[2] B. M. Escher, R. L. De Matos Filho, and L. Davidovich, General framework for estimating the ultimate precision limit in noisy quantum-enhanced metrology, Nature Physics 7, 406 (2011), arXiv:1201.1693.

[3] R. Demkowicz-Dobrzański, J. Kołodyński, and M. Guta, The elusive Heisenberg limit in quantum-enhanced metrology, Nature Communications 3, 1063 (2012), arXiv:1201.3940.

[4] J. Kołodyński and R. Demkowicz-Dobrzański, Efficient tools for quantum metrology with uncorrelated noise, New Journal of Physics 15, 073043 (2013), arXiv:1303.7271.

[5] A. Smirne, J. Kołodyński, S. F. Huelga, and
R. Demkowicz-Dobrzański, Ultimate Precision Limits for Noisy Frequency Estimation, Physical Review Letters 116, 120801 (2016), arXiv:1511.02708.

[6] H. Yuan and C. H. F. Fung, Quantum parameter estimation with general dynamics, npj Quantum Information 3, 14 (2017), arXiv:1506.01909.

[7] S. Zhou and L. Jiang, Asymptotic theory of quantum channel estimation, PRX Quantum 2, 010343 (2021), arXiv:2003.10559.

[8] E. M. Kessler, I. Lovchinsky, A. O. Sushkov, and M. D. Lukin, Quantum error correction for metrology, Physical Review Letters 112, 150802 (2014), arXiv:1310.3260.

[9] W. Dür, M. Skotiniotis, F. Fröwis, and B. Kraus, Improved quantum metrology using quantum error correction, Physical Review Letters 112, 080801 (2014), arXiv:1310.3750.

[10] R. Demkowicz-Dobrzański, J. Czajkowski, and P. Sekat- 
ski, Adaptive quantum metrology under general Markovian noise, Physical Review X 7, 041009 (2017), arXiv:1704.06280.

[11] S. Zhou, M. Zhang, J. Preskill, and L. Jiang, Achieving the Heisenberg limit in quantum metrology using quantum error correction, Nature Communications 9, 1 (2018), arXiv:1706.02445.

[12] Y. Matsuzaki, S. C. Benjamin, and J. Fitzsimons, Magnetic field sensing beyond the standard quantum limit under the effect of decoherence, Physical Review A 84, 012103 (2011), arXiv:1101.2561.

[13] A. W. Chin, S. F. Huelga, and M. B. Plenio, Quantum metrology in non-Markovian environments, Physical Review Letters 109, 233601 (2012), arXiv:1103.1219.

[14] K. Berrada, Non-Markovian effect on the precision of parameter estimation, Physical Review A 88, 035806 (2013).

[15] K. Macieszczak, Zeno limit in frequency estimation with non-Markovian environments, Physical Review A 92, 010102 (2015), arXiv:1403.1333.

[16] Y.S. Wang, C. Chen, and J. H. An, Quantum metrology in local dissipative environments, New Journal of Physics 19, 113019 (2017), arXiv:1702.07145.

[17] Y. Yang, Memory effects in quantum metrology, Physical Review Letters 123, 110501 (2019), arXiv:1904.07267.

[18] N. Mirkin, M. Larocca, and D. Wisniacki, Quantum metrology in a non-Markovian quantum evolution, Physical Review A 102, 022618 (2020), arXiv:1912.04675.

[19] G. Chiribella, G. M. D'Ariano, and P. Perinotti, Quantum circuit architecture, Physical Review Letters 101, 060401 (2008), arXiv:0712.1325.

[20] G. Chiribella, G. M. D'Ariano, and P. Perinotti, Transforming quantum operations: Quantum supermaps, EPL 83, 30004 (2008), arXiv:0804.0180.

[21] G. Chiribella, G. M. D'Ariano, and P. Perinotti, Theoretical framework for quantum networks, Physical Review A 80, 022339 (2009), arXiv:0904.4483.

[22] G. Chiribella, G. M. D'Ariano, and P. Perinotti, Memory effects in quantum channel discrimination, Physical Review Letters 101, 180501 (2008), arXiv:0803.3237.

[23] G. Chiribella and D. Ebler, Optimal quantum networks and one-shot entropies, New Journal of Physics 18, 093053 (2016), arXiv:1606.02394.

[24] P. Taranto, F. A. Pollock, S. Milz, M. Tomamichel, and K. Modi, Quantum Markov order, Physical Review Letters 122, 140401 (2019), arXiv:1805.11341.

[25] Q. Dong, M. T. Quintino, A. Soeda, and M. Murao, Success-or-Draw: A Strategy Allowing Repeat-UntilSuccess in Quantum Computation, Physical Review Letters 126, 150504 (2021), arXiv:2011.01055.

[26] J. Preskill, Quantum computing in the NISQ era and beyond, Quantum 2, 79 (2018), arXiv:1801.00862.

[27] M. D. Choi, Completely positive linear maps on complex matrices, Linear Algebra and Its Applications 10, 285 (1975).

[28] C. W. Helstrom, Journal of Statistical Physics (Springer, 1969).

[29] A. Holevo, Probabilistic and Statistical Aspects of Quantum Theory (Springer, 2011).

[30] A. Fujiwara and H. Imai, A fibre bundle over manifolds of quantum channels and its application to quantum statistics, Journal of Physics A: Mathematical and Theoretical 41, 255304 (2008).

[31] See Appendix for the proof of Theorem 1, extra numer- ical results, as well as a note on adaptive estimation of quantum channels, which includes which contains Refs. [32-44].

[32] J. S. Sidhu and P. Kok, Geometric Perspective on Quantum Parameter Estimation, AVS Quantum Science 2, 014701 (2020), arXiv:1907.06628.

[33] L. P. Hughston, R. Jozsa, and W. K. Wootters, A complete classification of quantum ensembles having a given density matrix, Physics Letters A 183, 14 (1993).

[34] R. T. Rockafellar, Convex analysis (Princeton University Press, 1977).

[35] G. Chiribella, Optimal networks for quantum metrology: Semidefinite programs and product rules, New Journal of Physics 14, 125008 (2012), arXiv:1207.6172.

[36] J. Watrous, The Theory of Quantum Information (Cambridge University Press, 2018).

[37] G. Gutoski, On a measure of distance for quantum strategies, Journal of Mathematical Physics 53, 032202 (2012), arXiv:1008.4636.

[38] A. Molina and J. Watrous, Hedging bets with correlated quantum strategies, Proceedings of the Royal Society A 468, 2614 (2012), arXiv:1104.1140.

[39] M. A. Nielsen and I. L. Chuang, Quantum computation and quantum information (Cambridge University Press, 2000).

[40] M. Ziman, P. Štelmachovič, and V. Bužek, Description of quantum dynamics of open systems based on collisionlike models, Open Systems and Information Dynamics 12, 81 (2005).

[41] O. Oreshkov and T. A. Brun, Continuous quantum error correction for non-Markovian decoherence, Physical Review A 76, 022318 (2007), arXiv:0705.2342.

[42] H. Krovi, O. Oreshkov, M. Ryazanov, and D. A. Lidar, Non-Markovian dynamics of a qubit coupled to an Ising spin bath, Physical Review A 76, 052117 (2007).

[43] M. C. Grant and S. P. Boyd, CVX: Matlab Software for Disciplined Convex Programming, Version 2.2 (2020).

[44] Comb estimation: gitlab.com/donion74/combestimation (2021).

[45] We remark that in reality the noise and the phase accumulation occur simultaneously rather than sequentially. However, when each step is short enough $(\tau, t \ll 1)$, the circuit model still yields a good approximation of practical noises (see, e.g., [46, 47]).

[46] F. Ciccarello, G. M. Palma, and V. Giovannetti, Collisionmodel-based approach to non-Markovian quantum dynamics, Physical Review A 87, 040103 (2013), arXiv:1207.6554.

[47] S. Kretschmer, K. Luoma, and W. T. Strunz, Collision model for non-Markovian quantum dynamics, Physical Review A 94, 012106 (2016), arXiv:1603.00408.

[48] W. Wu and C. Shi, Quantum parameter estimation in a dissipative environment, Physical Review A 102, 032607 (2020).

[49] A. V. Uvarov, A. S. Kardashin, and J. D. Biamonte, Machine learning phase transitions with a quantum processor, Physical Review A 102, 012415 (2020), arXiv:1906.10155.

[50] M. Szczykulska, T. Baumgratz, and A. Datta, Multiparameter quantum metrology, Advances in Physics: $X$ 1, 621 (2016).

[51] R. Demkowicz-Dobrzański, W. Górecki, and M. Guta, Multi-parameter estimation beyond quantum Fisher information, Journal of Physics A: Mathematical and The- 
oretical 53, 363001 (2020), arXiv:2001.11742.

[52] R. D. Gill and S. Massar, State estimation for large ensembles, Physical Review A 61, 042312 (2000).

[53] S. Ragy, M. Jarzyna, and R. Demkowicz-Dobrzański, Compatibility in multiparameter quantum metrology, Physical Review A 94, 052108 (2016), arXiv:1608.02634.

[54] M. Gessner, L. Pezzè, and A. Smerzi, Sensitivity Bounds for Multiparameter Quantum Metrology, Physical Review Letters 121, 130503 (2018), arXiv:1806.05665.

[55] F. Albarelli, J. F. Friel, and A. Datta, Evaluating the Holevo Cramér-Rao Bound for Multiparameter Quantum Metrology, Physical Review Letters 123, 200503 (2019),
arXiv:1906.05724.

[56] Y. Yang, G. Chiribella, and M. Hayashi, Attaining the Ultimate Precision Limit in Quantum State Estimation, Communications in Mathematical Physics 368, 223 (2019), arXiv:1802.07587.

[57] J. Suzuki, Y. Yang, and M. Hayashi, Quantum state estimation with nuisance parameters, Journal of Physics A: Mathematical and Theoretical 53, 453001 (2020), arXiv:1911.02790.

[58] A. Yurtsever, J. A. Tropp, O. Fercoq, M. Udell, and V. Cevher, Scalable Semidefinite Programming, SIAM Journal on Mathematics of Data Science 3, 171 (2021), arXiv:1912.02949.

\section{Appendix A: Proof of Theorem 1}

Here we prove Theorem 1 of the main text by expressing the QFI of a quantum comb as a semidefinite program and deriving its dual form. Note that we use a slightly different formulation of the primal problem that is useful for the numerical implementation. In Subsection A 1, we express the QFI of a parametrised comb as a semidefinite programm. In Subsection A 2, we derive the corresponding dual problem for $N>1$, in Subsection A 3, we treat the case $N=1$. In Subsection A 4, we handle the minimisation over equivalent decompositions, and in Subsection A 5, we show the changes needed to obtain the dual problem in terms of the conditional min-entropy.

\section{The comb QFI as an optimization problem}

In this subsection, we show that the QFI of quantum combs can be expressed as a semidefinite program. The derivation uses a variational expression for the QFI of quantum states [30, Theorem 1], which we introduce here as the following lemma.

Lemma 1. Let $\left\{\rho_{\theta} \mid \theta \in \Theta\right\}$ be a smooth curve of quantum states of constant rank $r$ and $q \geq r$ an arbitrary integer. The QFI of a state $\rho_{\theta}$ is

$$
J\left(\rho_{\theta}\right)=4 \min _{\left\{\left|\psi_{\theta, i}\right\rangle\right\}} \sum_{i=1}^{q} \operatorname{tr}\left[\left|\dot{\psi}_{\theta, i}\right\rangle\left\langle\dot{\psi}_{\theta, i}\right|\right]
$$

Here, the minimisation is over all $\left\{\left|\psi_{\theta, i}\right\rangle\right\}$ such that

$$
\rho_{\theta}=\sum_{i=1}^{q}\left|\psi_{\theta, i}\right\rangle\left\langle\psi_{\theta, i}\right|
$$

Now, consider a parametrised $\operatorname{comb} C_{\theta} \in \operatorname{Comb}\left[\left(\mathcal{H}_{1}, \mathcal{H}_{2}\right), \ldots,\left(\mathcal{H}_{2 N-1}, \mathcal{H}_{2 N}\right)\right]$ with $\theta \in \Theta$ and its QFI [Eq. (3) in the main text]

$$
J\left(C_{\theta}\right)=\max _{T} J\left(C_{\theta} * T\right)
$$

for $T \in \operatorname{Comb}\left[\left(\varnothing, \mathcal{H}_{1}\right), \ldots,\left(\mathcal{H}_{2 N-2}, \mathcal{H}_{2 N-1} \otimes \mathcal{H}_{\text {aux }}\right)\right]$. We split the derivation into multiple lemmata.

Lemma 2. The QFI of a comb $C_{\theta}$ can be expressed as

$$
J\left(C_{\theta}\right)=\max _{T} \min _{\left\{\left|C_{\theta, i}\right\rangle\right\}} \operatorname{tr}\left[T\left(\mathbb{1}_{\text {aux }} \otimes \operatorname{tr}_{2 N}\left[\Omega_{\theta}\right]\right)\right] .
$$

Proof. We first fix a probe $T$ and consider a decomposition

$$
C_{\theta}=\sum_{i=1}^{q}\left|C_{\theta, i}\right\rangle\left\langle C_{\theta, i}\right|
$$


with $q \geq \max \left\{\operatorname{rank}\left(C_{\theta}\right) \mid \theta \in \Theta\right\}$ components. Due to the linearity of the link product and the convexity of the QFI [32], the maximal QFI will be achieved for pure $T=|T\rangle\langle T|{ }^{1}$ Using the definition of the link product [Eq. (2) of the main text], we can write

$$
C_{\theta} * T=\sum_{i=1}^{q}\left|v_{\theta, i}\right\rangle\left\langle v_{\theta, i}\right|
$$

where $\left|v_{\theta, i}\right\rangle$ can be written in components of $\left|C_{\theta, i}\right\rangle$ and $|T\rangle$ as

$$
\left|v_{\theta, i}\right\rangle=\sum_{m_{1}, \ldots, m_{2 N}, m_{\mathrm{aux}}} C_{\theta, i}^{m_{1} \ldots m_{2 N-1} m_{2 N}} T^{m_{1} \ldots m_{2 N-1} m_{\mathrm{aux}}}\left|m_{2 N} m_{\mathrm{aux}}\right\rangle .
$$

Now, there arise some subtle issues regarding the existence of the QFI of a quantum comb that trace back to the existence of the QFI of a quantum state (Lemma 1). In particular, we assume:

1. The comb $C_{\theta}$ has a decomposition (A5) with number of components equal to $r:=\max \left\{\operatorname{rank}\left(C_{\theta}\right) \mid \theta \in \Theta\right\}$ such that each component $\left|C_{\theta, i}\right\rangle$ is continuously differentiable.

2. We assume that for a given probe $T$ the link product $C_{\theta} * T$ has constant rank for all $\theta \in \Theta$.

Under these conditions, for each probe $T$ the set $\left\{C_{\theta} * T \mid \theta \in \Theta\right\}$ is a smooth curve of quantum states with constant rank that immediately allows for application of Lemma 1. Applying Eq. (A1) to Eq. (A6), we find the QFI of the output to be

$$
J\left(C_{\theta} * T\right)=\min _{\left\{\left|C_{\theta, i}\right\rangle\right\}} \operatorname{tr}\left[T\left(\mathbb{1}_{\mathrm{aux}} \otimes \operatorname{tr}_{2 N}\left[\Omega_{\theta}\right]\right)\right],
$$

the minimisation is taken over all decompositions as in Eq. (A5) and we use the performance operator

$$
\Omega_{\theta}=4 \sum_{i=1}^{q}\left(\left|\dot{C}_{\theta, i}\right\rangle\left\langle\dot{C}_{\theta, i}\right|\right)^{T_{1 \ldots 2 N-1}} .
$$

Including the maximization over all probes, we obtain the desired result.

We now show how to deal with the minimization over equivalent decompositions.

Lemma 3. The QFI of a comb $C_{\theta}$ can be expressed as

$$
J\left(C_{\theta}\right)=\max _{T} \min _{h} \operatorname{tr}\left[T\left(\mathbb{1}_{\text {aux }} \otimes \operatorname{tr}_{2 N}\left[\Omega_{\theta}(h)\right]\right)\right] .
$$

Proof. Let us fix an ensemble decomposition $\left\{\left|C_{\theta, i}\right\rangle\right\}$ of size $r=\max \left\{\operatorname{rank}\left(C_{\theta}\right) \mid \theta \in \Theta\right\}$. We represent $\left|C_{\theta, i}\right\rangle$ as columns vectors of $A_{\theta}^{(0)}:=\left[\left|C_{\theta, 1}\right\rangle, \ldots,\left|C_{\theta, r}\right\rangle\right]$ and extend it to an ensemble decomposition of size $q \geq r$ by considering $A_{\theta}:=A_{\theta}^{(0)} I_{r, q}$ where $I_{r, q}=\left[\mathbb{1}_{r} \mid 0_{r, q-r}\right]$ and $0_{r, q-r}$ denotes the $r \times(q-r)$ zero matrix. Arbitrary ensemble decompositions with $q$ components can be related to $A_{\theta}$ via a unitary $V_{\theta}: \tilde{A}_{\theta}=A_{\theta} V_{\theta}{ }^{2}$. The performance operator can be written as $\Omega_{\theta}=4\left(\dot{\tilde{A}}_{\theta} \dot{\tilde{A}}_{\theta}^{\dagger}\right)^{T_{1} \ldots 2 N-1}$ and the expression in brackets evaluates to

$$
\dot{\tilde{A}}_{\theta} \dot{\tilde{A}}_{\theta}^{+}=\left(\dot{A}_{\theta} V_{\theta}+A_{\theta} \dot{V}_{\theta}\right)\left(V_{\theta}^{+} \dot{A}_{\theta}^{+}+\dot{V}_{\theta}^{+} A_{\theta}^{+}\right)=\dot{A}_{\theta} \dot{A}_{\theta}^{+}+A_{\theta} \dot{V}_{\theta} V_{\theta}^{+} \dot{A}_{\theta}^{+}-\dot{A}_{\theta} \dot{V}_{\theta} V_{\theta}^{+} A_{\theta}^{+}-A_{\theta} \dot{V}_{\theta} V_{\theta}^{+} \dot{V}_{\theta} V_{\theta}^{+} A_{\theta}^{+},
$$

\footnotetext{
${ }^{1}$ For general $T=\sum_{l} p_{l}\left|T_{l}\right\rangle\left\langle T_{l}\right|$, Eq. (A6) reads $C_{\theta} * T=$ $\sum_{l} p_{l} \sum_{i}\left|v_{\theta, i l}\right\rangle\left\langle v_{\theta, i l}\right|$ for an appropriate $\left|v_{\theta, i l}\right\rangle$ (compare Eq. (A7)). Convexity of the QFI implies $J\left(C_{\theta} * T\right) \leq \sum_{l} p_{l} J\left(\sum_{i}\left|v_{\theta, i l}\right\rangle\left\langle v_{\theta, i l}\right|\right)=$ $\sum_{l} p_{l} J\left(C_{\theta} *\left|T_{l}\right\rangle\left\langle T_{l}\right|\right)$. The maximal QFI is thus achieved for pure $T$.

${ }^{2}$ For arbitrary ensemble decompositions $\sum_{i=1}^{q}\left|A_{i}\right\rangle\left\langle A_{i}\right|$ and $\sum_{i=1}^{q}\left|B_{i}\right\rangle\left\langle B_{i}\right|$ of a density matrix $\rho$, consider the corresponding bipartite states $\left|\psi^{A / B}\right\rangle:=\sum_{i=1}^{q}\left|A_{i} / B_{i}\right\rangle|i\rangle$, where $\{|i\rangle\}$ is an
}

orthonormal basis. Since $\left|\psi^{A}\right\rangle\left\langle\psi^{A}\right|$ and $\left|\psi^{B}\right\rangle\left\langle\psi^{B}\right|$ are two purifications of the same density matrix, there exists a unitary $V$ such that $(I \otimes V)\left|\psi^{A}\right\rangle=\left|\psi^{B}\right\rangle$ (known as the HJW purification theorem [33]) and we get the desired relation between the two ensemble decompositions. The argument can be easily extended to $\rho$ with non-unit trace. 
where we use $V_{\theta}^{\dagger} V_{\theta}=\mathbb{1}=V_{\theta} V_{\theta}^{+}$and $V_{\theta} \dot{V}_{\theta}^{+}=-\dot{V}_{\theta} V_{\theta}^{+}$. Notice in the above expression that the dependency on $V_{\theta}$ enters only via the Hermitian matrix $h:=i \dot{V}_{\theta} V_{\theta}^{+}$. Equivalently we can write

$$
\dot{\tilde{A}}_{\theta} \dot{\tilde{A}}_{\theta}^{\dagger}=\left(\dot{A}_{\theta}-i A_{\theta} h\right)\left(\dot{A}_{\theta}-i A_{\theta} h\right)^{\dagger} .
$$

It is thus sufficient to consider derivatives of the form

$$
\left|\dot{\tilde{C}}_{\theta, j}\right\rangle=\left|\dot{C}_{\theta, j}\right\rangle-i \sum_{j=1}^{q} h_{j i}\left|C_{\theta, i}\right\rangle
$$

and we write

$$
\Omega_{\theta}(h)=4 \sum_{j=1}^{q}\left(\left|\dot{\tilde{C}}_{\theta, j}\right\rangle\left\langle\dot{\tilde{C}}_{\theta, j}\right|\right)^{T_{1} \ldots 2 N-1}
$$

for the corresponding performance operator. The QFI can then be expressed as

$$
J\left(C_{\theta}\right)=\max _{T} \min _{h} \operatorname{tr}\left[T\left(\mathbb{1}_{\mathrm{aux}} \otimes \operatorname{tr}_{2 N}\left[\Omega_{\theta}(h)\right]\right)\right] .
$$

Next, we show that it is sufficient to consider Hermitian matrices of size $r \times r$.

Lemma 4. The minimum over $h$ in Eq. (A15) is achieved for a $r \times r$ Hermitian.

Proof. We insert $A_{\theta}=A_{\theta}^{(0)} I_{r, q}$ into Eq. (A12) and find

$$
\dot{\tilde{A}}_{\theta} \dot{\tilde{A}}_{\theta}^{\dagger}=\dot{A}_{\theta}^{(0)} I_{r, q} I_{r, q}^{\dagger}\left(\dot{A}_{\theta}^{(0)}\right)^{\dagger}-i A_{\theta}^{(0)} I_{r, q} h I_{r, q}^{\dagger}\left(\dot{A}_{\theta}^{(0)}\right)^{\dagger}+i \dot{A}_{\theta}^{(0)} I_{r, q} h I_{r, q}^{\dagger}\left(A_{\theta}^{(0)}\right)^{\dagger}+A_{\theta}^{(0)} I_{r, q} h^{2} I_{r, q}\left(A_{\theta}^{(0)}\right)^{\dagger},
$$

We decompose

$$
h=\left(\begin{array}{cc}
h_{r} & h_{2} \\
h_{2}^{\dagger} & h_{q-r}
\end{array}\right)
$$

into a $r \times r$ Hermitian $h_{r}$, a $(q-r) \times(q-r)$ Hermitian $h_{q-r}$ and a $r \times(q-r)$ matrix $h_{2}$. We note that $I_{r, q} I_{r, q}^{+}=\mathbb{1}_{r}$, $I_{r, q} h I_{r, q}^{+}=h_{r}, I_{r, q} h^{2} I_{r, q}^{+}=h_{r}^{2}+h_{2} h_{2}^{\dagger}$ and thus

$$
\begin{aligned}
\dot{\tilde{A}}_{\theta} \dot{\tilde{A}}_{\theta}^{\dagger} & =\dot{A}_{\theta}^{(0)}\left(\dot{A}_{\theta}^{(0)}\right)^{\dagger}-i A_{\theta}^{(0)} h_{r}\left(\dot{A}_{\theta}^{(0)}\right)^{\dagger}+i \dot{A}_{\theta}^{(0)} h_{r}\left(A_{\theta}^{(0)}\right)^{\dagger}+A_{\theta}^{(0)}\left(h_{r}^{2}+h_{2} h_{2}^{\dagger}\right)\left(A_{\theta}^{(0)}\right)^{\dagger} \\
& =\left(\dot{A}_{\theta}^{(0)}-i A_{\theta}^{(0)} h_{r}\right)\left(\dot{A}_{\theta}^{(0)}-i A_{\theta}^{(0)} h_{r}\right)^{\dagger}+A_{\theta}^{(0)} h_{2} h_{2}^{\dagger}\left(A_{\theta}^{(0)}\right)^{\dagger} .
\end{aligned}
$$

The last term $A_{\theta}^{(0)} h_{2} h_{2}^{\dagger}\left(A_{\theta}^{(0)}\right)^{\dagger}$ is positive semidefinite. If we denote by $\Omega_{\theta}(h)$ the performance operator that correponds to the ensemble decomposition $\tilde{A}_{\theta}$ and by $\Omega_{\theta}\left(h_{r}\right):=4\left(\dot{A}_{\theta}^{(0)}-i A_{\theta}^{(0)} h_{r}\right)\left(\dot{A}_{\theta}^{(0)}-i A_{\theta}^{(0)} h_{r}\right)^{\dagger}$ the performance operator of an ensemble decomposition of size $r$, we note that

$$
\Omega_{\theta}(h) \succeq \Omega_{\theta}\left(h_{r}\right) .
$$

Since Eq. (A15) is linear in $\Omega_{\theta}(h)$, it follows that the minimum over equivalent decompositions is achieved for a decomposition of size $r$.

In order to simplify the problem, we are going to exchange the minimisation and maximisation. We are allowed to do so since the function we are interested in is convex in $h$ and concave in $T$ [34]:

$$
J\left(C_{\theta}\right)=\min _{h} \max _{T} \operatorname{tr}\left[T\left(\mathbb{1}_{\text {aux }} \otimes \operatorname{tr}_{2 N}\left[\Omega_{\theta}\right]\right)\right] .
$$


We first fix $h$ and maximise over all probes. The problem can be stated as

$$
\text { maximise } \begin{aligned}
& \operatorname{tr}\left[T\left(\mathbb{1}_{\mathrm{aux}} \otimes \operatorname{tr}_{2 N}\left[\Omega_{\theta}(h)\right]\right)\right], \\
& \operatorname{tr}_{2 N-1, \text { aux }}[T]=\mathbb{1}_{2 N-2} \otimes T^{(N-1)}, \\
& \operatorname{tr}_{2 k-1}\left[T^{(k)}\right]=\mathbb{1}_{2 k-2} \otimes T^{(k-1)}, \quad k=2, \ldots, N-1, \\
& \operatorname{tr}_{1}\left[T^{(1)}\right]=1,
\end{aligned}
$$

where $T, T^{(k)}$ for $k=1, \ldots, N-1$ are positive semidefinite and the equality constraints arise from the definition of the comb [Eq. (1) of the main text]. Since the objective function and equality constraints are linear in $T$, this is a semidefinite program, which has efficient numerical methods. In addition, we can convert the comb QFI into a minimization problem using (strong) duality.

\section{Dual problem for $N>1$}

In this section, we are going to derive the dual problem of the primal problem Eq. (A22) for $N>1$.

Lemma 5. The dual problem of the primal problem Eq. (A22) for $N>1$ is given as

$$
\begin{aligned}
& \text { minimize } S^{(0)} \text {, } \\
& \text { subject to } \quad \mathbb{1}_{1} S^{(0)} \succeq \operatorname{tr}_{2}\left[S^{(1)}\right] \text {, } \\
& \mathbb{1}_{2 k-1} \otimes S^{(k-1)} \succeq \operatorname{tr}_{2 k}\left[S^{(k)}\right], \quad k=2, \ldots, N-1 \\
& \mathbb{1}_{2 N-1} \otimes S^{(N-1)} \succeq \operatorname{tr}_{2 N}\left[\Omega_{\theta}(h)\right] .
\end{aligned}
$$

In addition, strong duality holds, that is the solution of the dual problem coincides with the solution of the primal problem.

Proof. We follow the approach of Ref. [35] and cast the primal problem in the standard form:

$$
\begin{array}{lrl}
\text { maximize } & \operatorname{tr}[\mathfrak{T} \mathfrak{C}] \\
\text { subject to } & \mathrm{L}(\mathfrak{T})=\mathfrak{O} \\
\mathfrak{T} \succeq 0
\end{array}
$$

where we compose the following quantities:

$$
\begin{aligned}
& \mathfrak{T}=\left(\bigoplus_{k=1}^{N-1} T^{(k)}\right) \oplus T, \quad \mathrm{~L}(\mathfrak{T})=\bigoplus_{k=1}^{N} L^{(k)}, \\
& \mathfrak{C}=\left(\bigoplus_{k=1}^{N-1} 0^{(k)}\right) \oplus\left(\mathbb{1}_{\mathrm{aux}} \otimes \operatorname{tr}_{2 N}\left[\Omega_{\theta}(h)\right]\right), \\
& \mathfrak{O}=1 \oplus\left(\bigoplus_{k=2}^{N} 0^{(k)}\right) .
\end{aligned}
$$

Here, $0^{(k)}$ denotes the zero matrix on an appropriately chosen space. By composing these block matrices, we can transform the constraints on the probe in one single constraint. Furthermore, we decompose

$$
\begin{aligned}
& L^{(1)}=\operatorname{tr}_{1}\left[T^{(1)}\right] \\
& L^{(k)}=\operatorname{tr}_{2 k-1}\left[T^{(k)}\right]-\mathbb{1}_{2 k-2} \otimes T^{(k-1)}, \quad \text { for } k=2, \ldots, N-1, \\
& L^{(N)}=\operatorname{tr}_{2 N-1, \text { aux }}[T]-\mathbb{1}_{2 N-2} \otimes T^{(N-1)} .
\end{aligned}
$$

The dual problem of the problem Eq. (A24) is given as [36]

$$
\begin{array}{lr}
\text { minimize } & \operatorname{tr}[\mathfrak{S O}] \\
\text { subject to } & \mathrm{L}^{+}(\mathfrak{S}) \succeq \mathfrak{C}, \\
& \mathfrak{S} \text { Hermitian, }
\end{array}
$$


where we decompose

$$
\mathfrak{S}=\bigoplus_{k=0}^{N-1} S^{(k)}, \quad L^{\dagger}(\mathfrak{S})=\bigoplus_{k=1}^{N}\left(L^{\dagger}\right)^{(k)},
$$

and $\mathrm{L}^{\dagger}$ is the dual map with respect to the Hilbert-Schmidt product: It satisfies $\operatorname{tr}[\mathfrak{S L}(\mathfrak{T})]=\operatorname{tr}\left[\mathrm{L}^{\dagger}(\mathfrak{S}) \mathfrak{T}\right]$ for all $\mathfrak{S}, \mathfrak{T}$. Using this definition, we find for $N>1$

$$
\begin{aligned}
\left(L^{\dagger}\right)^{(1)} & =\mathbb{1}_{1} S^{(0)}-\operatorname{tr}_{2}\left[S^{(1)}\right], \\
\left(L^{\dagger}\right)^{(k)} & =\mathbb{1}_{2 k-1} \otimes S^{(k-1)}-\operatorname{tr}_{2 k}\left[S^{(k)}\right] \quad \text { for } k=2, \ldots, N-1, \\
\left(L^{\dagger}\right)^{(N)} & =\mathbb{1}_{2 N-1, \text { aux }} \otimes S^{(N-1)} .
\end{aligned}
$$

Inserting these decompositions into Eq. (A27), we obtain

$$
\begin{aligned}
& \text { minimize } S^{(0)} \text {, } \\
& \text { subject to } \quad \mathbb{1}_{1} S^{(0)} \succeq \operatorname{tr}_{2}\left[S^{(1)}\right] \text {, } \\
& \mathbb{1}_{2 k-1} \otimes S^{(k-1)} \succeq \operatorname{tr}_{2 k}\left[S^{(k)}\right], \quad k=2, \ldots, N-1 \\
& \mathbb{1}_{2 N-1, \text { aux }} \otimes S^{(N-1)} \succeq \mathbb{1}_{\text {aux }} \otimes \operatorname{tr}_{2 N}\left[\Omega_{\theta}(h)\right] .
\end{aligned}
$$

The last constraint is equivalent to $\mathbb{1}_{2 N-1} \otimes S^{(N-1)} \succeq \operatorname{tr}_{2 N}\left[\Omega_{\theta}(h)\right]$.

Note that $S^{(N-1)} \succeq 0$ since $\Omega_{\theta}(h) \succeq 0$. This implies recursively that $S^{(k)} \succeq 0$ for all $k=0,1, \ldots, N-1$. In addition, there exists an $\mathfrak{S}$ with $\mathrm{L}^{\dagger}(\mathfrak{S}) \succ \mathfrak{C}$. For example, we can set recursively

$$
\begin{aligned}
S^{(N-1)} & =2\left\|\operatorname{tr}_{2 N}\left[\Omega_{\theta}(h)\right]\right\|_{\infty} \mathbb{1}_{1,2, \ldots, 2 N-2,} \\
S^{(k)} & =2 \operatorname{tr}_{2 k+1,2 k+2}\left[S^{(k+1)}\right], \quad k=0, \ldots, N-2 .
\end{aligned}
$$

This implies, together with the fact that the QFI is upper-bounded [17, Theorem 1], that the assumptions of Slater's theorem on strong duality $[37,38]$ are satisfied.

The following lemma shows that we can turn the inequality constraints into equality constraints.

Lemma 6. For every $\mathfrak{S}$ that satisfies the bounds in the dual problem Eq. (A23), we can construct a $\widetilde{\mathfrak{S}}=\bigoplus_{k=0}^{N-1} \tilde{S}^{(k)}$ such that the first $(N-1)$ inequalities become equalities and the dual form reads

$$
\begin{aligned}
\text { minimize } & \tilde{S}^{(0)}, \\
\text { subject to } & \mathbb{1}_{1} \tilde{S}^{(0)}=\operatorname{tr}_{2}\left[\tilde{S}^{(1)}\right], \\
& \mathbb{1}_{2 k-1} \otimes \tilde{S}^{(k-1)}=\operatorname{tr}_{2 k}\left[\tilde{S}^{(k)}\right], \quad k=2, \ldots, N-1 \\
\mathbb{1}_{2 N-1} \otimes \tilde{S}^{(N-1)} & \succeq \operatorname{tr}_{2 N}\left[\Omega_{\theta}(h)\right]
\end{aligned}
$$

Proof. We prove the lemma by induction. Assume that $\mathfrak{S}$ satisfies the constraints Eq. (A23). Set

$$
\begin{aligned}
& \tilde{S}^{(0)}=S^{(0)}, \\
& \delta^{(1)}=\mathbb{1}_{1} \tilde{S}^{(0)}-\operatorname{tr}_{2}\left[S^{(1)}\right] \succeq 0, \\
& \tilde{S}^{(1)}=S^{(1)}+\delta^{(1)} \otimes \rho_{2}, \\
& \tilde{S}^{(j)}=S^{(j)}, \quad j=2, \ldots, N-1,
\end{aligned}
$$

where $\rho_{2}$ is an arbitrary quantum state in $\mathcal{S}\left(\mathcal{H}_{2}\right)$. It follows that $\operatorname{tr}_{2}\left[\tilde{S}^{(1)}\right]=\mathbb{1}_{1} \tilde{S}^{(0)}$, hence $\tilde{\mathcal{S}}$ achieves equality in the first constraint. Moreover, since $\delta^{(1)} \succeq 0$, we have $\mathbb{1}_{3} \otimes \tilde{S}^{(1)} \succeq \mathbb{1}_{3} \otimes S^{(1)}$ and since $S^{(1)}$ satisfies the second constraint, 
it holds that $\mathbb{1}_{3} \otimes S^{(1)} \succeq \operatorname{tr}_{4}\left[S^{(2)}\right]=\operatorname{tr}_{4}\left[\tilde{S}^{(2)}\right]$. Hence, $\mathbb{1}_{3} \otimes \tilde{S}^{(1)} \succeq \operatorname{tr}_{4}\left[\tilde{S}^{(2)}\right]$ and $\tilde{S}^{(1)}$ satisfies the second constraint. In total, $\widetilde{\mathfrak{S}}$ has the same objective value as $\mathfrak{S}$, satisfies all constraints and achieves equality in the first constraint.

Now, assume that $\mathfrak{S}$ achieves equality in the first $1 \leq k \leq N-2$ constraints and define

$$
\begin{aligned}
\tilde{S}^{(j)} & =S^{(j)}, \quad j=1, \ldots, k, \\
\delta^{(k+1)} & =\mathbb{1}_{2 k+1} \otimes \tilde{S}^{(k)}-\operatorname{tr}_{2 k+2}\left[S^{(k+1)}\right] \succeq 0, \\
\tilde{S}^{(k+1)} & =S^{(k+1)}+\delta^{(k+1)} \otimes \rho_{2 k \prime} \\
\tilde{S}^{(j)} & =S^{(j)}, \quad j=k+2, \ldots, N-1,
\end{aligned}
$$

where $\rho_{2 k}$ is an arbitrary quantum state on $\mathcal{S}\left(\mathcal{H}_{2 k}\right)$. Similar to before, $\widetilde{\mathfrak{S}}$ achieves the same objective value as $\mathfrak{S}$, satisfies all constraints and achieves equality in the first $(k+1)$ constraints. By induction, it follows that for every $\mathfrak{S}$ satisfying the constraints, there exists $\mathfrak{S}$ achieving the same objective value and satisfying the first $(N-1)$ constraints with equality.

In summary, we have found the dual problem

$$
\begin{aligned}
& \text { minimize } \quad S^{(0)} \text {, } \\
& \text { subject to } \quad \mathbb{1}_{1} S^{(0)}=\operatorname{tr}_{2}\left[S^{(1)}\right] \text {, } \\
& \mathbb{1}_{2 k-1} \otimes S^{(k-1)}=\operatorname{tr}_{2 k}\left[S^{(k)}\right], \quad k=2, \ldots, N-1, \\
& \mathbb{1}_{2 N-1} \otimes S^{(N-1)} \succeq \operatorname{tr}_{2 N}\left[\Omega_{\theta}(h)\right]
\end{aligned}
$$

and shown that its solution is equal to the QFI of the $\operatorname{comb} C_{\theta}$. Note that the first two equality constraints imply that $S^{(N-1)} / S^{(0)}$ is a quantum comb in $\operatorname{Comb}\left[\left(\mathcal{H}_{1}, \mathcal{H}_{2}\right), \ldots,\left(\mathcal{H}_{2 N-3}, \mathcal{H}_{2 N-2}\right)\right]$. Thus, by setting $\lambda=S^{(0)}$ and $S_{\text {nor }}^{(k)}=\frac{S^{(k)}}{\lambda}$ for $k=1, \ldots, N-1$, the dual problem corresponds to:

$$
\begin{aligned}
& \text { minimise } \\
& \lambda \\
& \text { subject to } \quad \mathbb{1}_{1}=\operatorname{tr}_{2}\left[S_{\text {nor }}^{(1)}\right] \text {, } \\
& \mathbb{1}_{2 k-1} \otimes S_{\text {nor }}^{(k-1)}=\operatorname{tr}_{2 k}\left[S_{\text {nor }}^{(k)}\right], \quad k=2, \ldots, N-1, \\
& \mathbb{1}_{2 N-1} \otimes \lambda S_{\text {nor }}^{(N-1)} \succeq \operatorname{tr}_{2 N}\left[\Omega_{\theta}(h)\right] .
\end{aligned}
$$

By redefining $S^{(k)}:=S_{\text {nor }}^{(k)}$ we recover the problem in Algorithm 1 without minimising over equivalent decompositions.

\section{Dual problem for $N=1$}

In this subsection, we derive the dual problem for the case $N=1$, i.e., when the comb is a quantum channel.

Lemma 7. The comb QFI for $N=1$ is given by

$$
J\left(C_{\theta}\right)=\min _{h}\left\|\operatorname{tr}_{2}\left[\Omega_{\theta}(h)\right]\right\|_{\infty} .
$$

This agrees with a result of Ref. [30, Theorem 4].

Proof. First we start with the primal problem Eq. (A22) and cast it into the standard form Eq. (A24)

$$
\begin{array}{lr}
\text { maximize } & \operatorname{tr}[\mathfrak{T} \mathfrak{C}], \\
\text { subject to } & \mathrm{L}(\mathfrak{T})=\mathfrak{O}, \\
& \mathfrak{T} \succeq 0,
\end{array}
$$

where

$$
\mathfrak{T}=T, \quad \mathfrak{C}=\mathbb{1}_{\mathrm{aux}} \otimes \operatorname{tr}_{2}\left[\Omega_{\theta}(h)\right], \quad \mathrm{L}(\mathfrak{T})=\operatorname{tr}_{\mathrm{aux}, 1}[T], \quad \mathfrak{O}=1 .
$$


These definitions coincide with the ones made for $N>1$. The dual problem takes the form

$$
\begin{array}{lr}
\text { minimize } & \operatorname{tr}[\mathfrak{S O},] \\
\text { subject to } & \mathrm{L}^{\dagger}(\mathfrak{S}) \succeq \mathfrak{C},
\end{array}
$$

where we compose $\mathfrak{S}=S^{(0)}, \mathrm{L}^{\dagger}(\mathfrak{S})=\left(L^{\dagger}\right)^{(1)}$. Now

$$
\begin{aligned}
\operatorname{tr}[\mathrm{L}(\mathfrak{T}) \mathfrak{S}] & =\operatorname{tr}_{\mathrm{aux}, 1}[T] S^{(0)}, \\
\operatorname{tr}\left[\mathrm{L}^{\dagger}(\mathfrak{S}) \mathfrak{T}\right] & =\operatorname{tr}_{\mathrm{aux}, 1}\left[\left(L^{\dagger}\right)^{(1)} T\right] .
\end{aligned}
$$

We conclude that $\left(L^{\dagger}\right)^{(1)}=S^{(0)} \mathbb{1}_{\mathrm{aux}, 1}$ and thus by setting $\lambda:=S^{(0)}$ the dual problem takes the form

$$
\begin{array}{cc}
\text { minimize } & \lambda, \\
\text { subject to } & \lambda \mathbb{1}_{\text {aux }, 1} \succeq \mathbb{1}_{\text {aux }} \otimes \operatorname{tr}_{2}\left[\Omega_{\theta}(h)\right] .
\end{array}
$$

We note that the last constraint is equivalent to $\lambda \mathbb{1}_{1} \succeq \operatorname{tr}_{2}\left[\Omega_{\theta}(h)\right]$ and the minimal $\lambda$ is equal to $\left\|\operatorname{tr}_{2}\left[\Omega_{\theta}(h)\right]\right\|_{\infty}$. Taking into account the minimization over Hermitian matrices, we find

$$
J\left(C_{\theta}\right)=\min _{h}\left\|\operatorname{tr}_{2}\left[\Omega_{\theta}(h)\right]\right\|_{\infty} .
$$

Using the relation between ensemble decomposition $\left|C_{\theta, i}\right\rangle$ and Kraus operators $\hat{C}_{\theta, i}$

$$
\left|C_{\theta, i}\right\rangle=\sum_{k_{2}=1}^{\operatorname{dim} \mathcal{H}_{2}}\left(\hat{C}_{i}^{T} \otimes \mathbb{1}_{2}\right)\left(\left|k_{2}\right\rangle \otimes\left|k_{2}\right\rangle\right),
$$

we can evaluate for a fixed $h=0$

$$
\operatorname{tr}_{2}\left[\Omega_{\theta}(h=0)\right]=4 \operatorname{tr}_{2}\left[\sum_{i}\left(\left|\dot{C}_{\theta, i}\right\rangle\left\langle\dot{C}_{\theta, i}\right|\right]^{T}=4 \sum_{i} \dot{\hat{C}}_{\theta, i}^{+} \dot{\hat{C}}_{\theta, i} .\right.
$$

Taking into account the minimisation over equivalent Kraus operators we get the QFI of quantum channels, first obtained by Fujiwara and Imai in Ref. [30]

$$
J\left(C_{\theta}\right)=4 \min _{\left\{\hat{C}_{\theta, i}\right\}}\left\|\sum_{i} \dot{\hat{C}}_{\theta, i}^{+} \dot{\hat{C}}_{\theta, i}\right\|_{\infty} .
$$

\section{Equivalent decompositions}

In this subsection, we show how to let the matrix $h$ enter linearly in the dual problem. In Eq. (A36), we replace $\lambda$ by $4 \lambda$ and obtain the problem

$$
\begin{aligned}
& \text { minimise } \\
& 4 \lambda \\
& \text { subject to } \\
& \operatorname{tr}_{2}\left[S^{(1)}\right]=\mathbb{1}_{1}, \\
& \operatorname{tr}_{2 k}\left[S^{(k)}\right]=\mathbb{1}_{2 k-1} \otimes S^{(k-1)}, \quad k=2, \ldots, N-1, \\
& \mathbb{1}_{2 N-1} \otimes \lambda S^{(N-1)} \succeq \frac{1}{4} \operatorname{tr}_{2 N}\left[\Omega_{\theta}(h)\right] .
\end{aligned}
$$

For a given decomposition $\left\{\left|C_{\theta, i}\right\rangle\right\}$, we define $\left|c_{i, m_{2 N}}\right\rangle=\sum_{m_{1} \ldots m_{2 N-1}} \dot{\tilde{C}}_{i, \theta}^{m_{1} \ldots m_{2 N-1} m_{2 N}}\left|m_{1} \ldots m_{2 N-1}\right\rangle(\bar{a}$ denotes the complex conjugate of $a$ ) and set up

$$
A(h, \lambda, S)=\left(\begin{array}{ccc|c} 
& & & \left\langle c_{1,1}\right| \\
& & & \vdots \\
& \mathbb{1}_{q \cdot d_{2 N}} & & \left\langle c_{q, d_{2 N}}\right| \\
\hline & & & \left|c_{q, d_{2 N}}\right\rangle \\
\left.\hline c_{1,1}\right\rangle & \ldots & \mathbb{1}_{2 N-1} \otimes \lambda S
\end{array}\right),
$$


with $q$ the number of components in the ensemble decomposition and $d_{2 N}=\operatorname{dim}\left(\mathcal{H}_{2 N}\right)$. By Schur's complement condition, positive semidefiniteness of $A$ is equivalent to

$$
\mathbb{1}_{2 N-1} \otimes \lambda S \succeq \sum_{i=1}^{q} \sum_{m=1}^{d_{2 N}}\left|c_{i, m}\right\rangle\left\langle c_{i, m}\right|=\frac{1}{4} \operatorname{tr}_{2 N}\left[\Omega_{\theta}(h)\right] .
$$

We can thus replace the last condition in Eq. (A47) and find

$$
\begin{aligned}
& \text { minimise } \\
& 4 \lambda \\
& \text { subject to } \\
& \operatorname{tr}_{2}\left[S^{(1)}\right]=\mathbb{1}_{1}, \\
& \operatorname{tr}_{2 k}\left[S^{(k)}\right]=\mathbb{1}_{2 k-1} \otimes S^{(k-1)}, \quad k=2, \ldots, N-1, \\
& A\left(h, \lambda, S^{(N-1)}\right) \succeq 0 .
\end{aligned}
$$

We find that this agrees with Algorithm 1. Equation (A13) implies that the variable $h$ enters linearly in Eq. (A48). Therefore the minimisation over equivalent decompositions is a semidefinite program.

\section{Dual problem in terms of min-entropy}

In this subsection, we show the derivation of Theorem 1 [Eq. (8) in the main text]. We start analogously to subsection A 2 with Eq. (A22), but want to

$$
\begin{array}{ll}
\text { maximise } & \operatorname{tr}\left[\left(\mathbb{1}_{2 N} \otimes T\right)\left(\mathbb{1}_{\text {aux }} \otimes \Omega_{\theta}(h)\right)\right], \\
\text { subject to } & T \in \operatorname{Comb}\left[\left(\varnothing, \mathcal{H}_{1}\right), \ldots,\left(\mathcal{H}_{2 N-2}, \mathcal{H}_{2 N-1} \otimes \mathcal{H}_{\text {aux }}\right)\right],
\end{array}
$$

that is we do not trace out over $\mathcal{H}_{2 N}$. We proceed analogously to Eq. (A26), but adjust

$$
L^{(N)}=\frac{1}{d_{2 N}} \operatorname{tr}_{2 N-1,2 N, \text { aux }}\left[\mathbb{1}_{2 N} \otimes T\right]-\mathbb{1}_{2 N-2} \otimes T^{(N-1)},
$$

with $d_{2 N}=\operatorname{dim}\left(\mathcal{H}_{2 N}\right)$. This in turn changes the dual map $\mathrm{L}^{\dagger}$ (Eq. (A29)) to

$$
\left(L^{\dagger}\right)^{(N)}=\frac{1}{d_{2 N}} \mathbb{1}_{2 N-1,2 N, \text { aux }} \otimes S^{(N-1)} .
$$

The dual problem Eq. (A36) is only affected in the last constraint, and becomes

$$
\begin{array}{ll}
\text { minimise } & \lambda \\
\text { subject to } & S \in \operatorname{Comb}\left[\left(\mathcal{H}_{1}, \mathcal{H}_{2}\right), \ldots,\left(\mathcal{H}_{2 N-3}, \mathcal{H}_{2 N-2}\right)\right], \\
& \frac{1}{d_{2 N}} \mathbb{1}_{2 N-1,2 N} \otimes \lambda S \succeq \Omega_{\theta}(h) .
\end{array}
$$

Comparing with the definition of the conditional min-entropy, we see that the minimal $\lambda$ satisfying the constraints is equal to

$$
d_{2 N} 2^{-H_{\min }(N \mid[N-1])_{\Omega_{\theta}(h)},}
$$

which agrees with Theorem 1.

\section{Appendix B: Frequency estimation under different types of noise}

In this section, we provide more details about the collision model used for the numerical implementation. In Subsection B 1, we elaborate on the different scenarios (non-Markovian vs. Markovian, with vs. without feedback), in Subsection B 2, we consider different interaction unitaries, in Subsection B3, we give some numerical results. 


\section{Scenarios}

In the following paragraph, we find a comb depending on how we control the system or whether we assume a Markovian or non-Markovian process. The different scenarios are depicted in Figure 7 and we assume that we are given the single-step unitary $U=U_{\text {int }}(\tau)\left(\mathbb{1}_{E} \otimes e^{-i H t}\right)$.

\section{a. Non-Markovian case with control}

We can depict the scenario where we are allowed to perform control operations between the interactions in Figure $7(a)$. The resulting Choi operator of the comb is obtained by tensoring $N$ times the system's degrees of freedom and perform matrix multiplication on the environmental degrees of freedom, resulting in an operator $U_{\text {tot }} \in \mathcal{L}\left(\mathcal{H}_{E} \otimes\right.$ $\left.\bigotimes_{i=1}^{2 N} \mathcal{H}_{i}\right)$. We initialise the first environment in $|0\rangle$ and trace out over the last environment $\mathcal{H}_{E_{2 N}}$. In order to obtain a vector, we flatten this matrix to a vector and denote this operation by vec. This results in the ensemble decomposition

$$
\left|C_{\theta, i}\right\rangle=\operatorname{vec}\left(\left\langle i\left|U_{\text {tot }}\right| 0\right\rangle\right),
$$

where $|i\rangle$ denotes an orthonormal basis of the last environmental system $\mathcal{H}_{E_{2 N}}$. We note that, if we include the environment, the evolution can be described by a unitary, and the ensemble decomposition results of our ignorance about the state of the environment. Note that the above expression resembles the expression for the Kraus operator $\hat{E}_{i}$ of a channel $\mathcal{E}(\rho)=\sum_{i} \hat{E}_{i} \rho \hat{E}_{i}^{\dagger}$, which can be obtained by its Stinespring dilation unitary $U$ in the following way: $\hat{E}_{i}=\langle i|U| 0\rangle$, where $|0\rangle$ denotes the initial state of the environment and $|i\rangle$ is an orthonormal basis of the environment [39].

\section{b. Non-Markovian case without control}

If we cannot perform any control operations, we have the scenario depicted in Figure 7(b). The total unitary $U_{\text {tot }}=U^{N}$ is the $N$-fold concatenation of $U$ resulting in an ensemble decomposition

$$
\left|C_{\theta, i}\right\rangle=\operatorname{vec}\left(\left\langle i\left|U^{N}\right| 0\right\rangle\right),
$$

where as before we flatten the matrix to a vector.

\section{c. Markovian case with control}

We assume the environment to have no memory. Hence, after each interaction, we trace over the environment and reinitialise it in the next step in the ground state, see Figure 7(c) for the $N=2$ case. For each interaction step $k=1, \ldots, N$, we obtain the components $\left|C_{\theta, i_{k}}\right\rangle=\operatorname{vec}\left(\left\langle i_{k}|U| 0\right\rangle\right)$ in the ensemble decomposition with $\left|i_{k}\right\rangle$ an orthonormal basis of the environment $\mathcal{H}_{E_{k}}$. In the end, we tensor these components to obtain the total ensemble decomposition

$$
\left|C_{\theta, \vec{i}=\left(i_{1}, \ldots, i_{N}\right)}\right\rangle=\bigotimes_{k=1}^{N}\left|C_{\theta, i_{k}}\right\rangle .
$$

\section{d. Markovian case without control}

In the Markovian case without control, we trace out the environment after each interaction and reinitialise it in the ground state and thus obtain components $U_{\theta, i_{k}}=\left\langle i_{k}|U| 0\right\rangle$ for each interaction $k=1, \ldots, N$. In addition, we do not perform any operation on the system's side. This scenario is depicted in Figure 7(d). We obtain the resulting ensemble decomposition by concatenating the components $C_{\theta, i_{k}}$ :

$$
\left|C_{\theta, \vec{i}=\left(i_{1}, \ldots, i_{N}\right)}\right\rangle=\operatorname{vec}\left(\prod_{k=1}^{N} U_{\theta, i_{k}}\right) .
$$




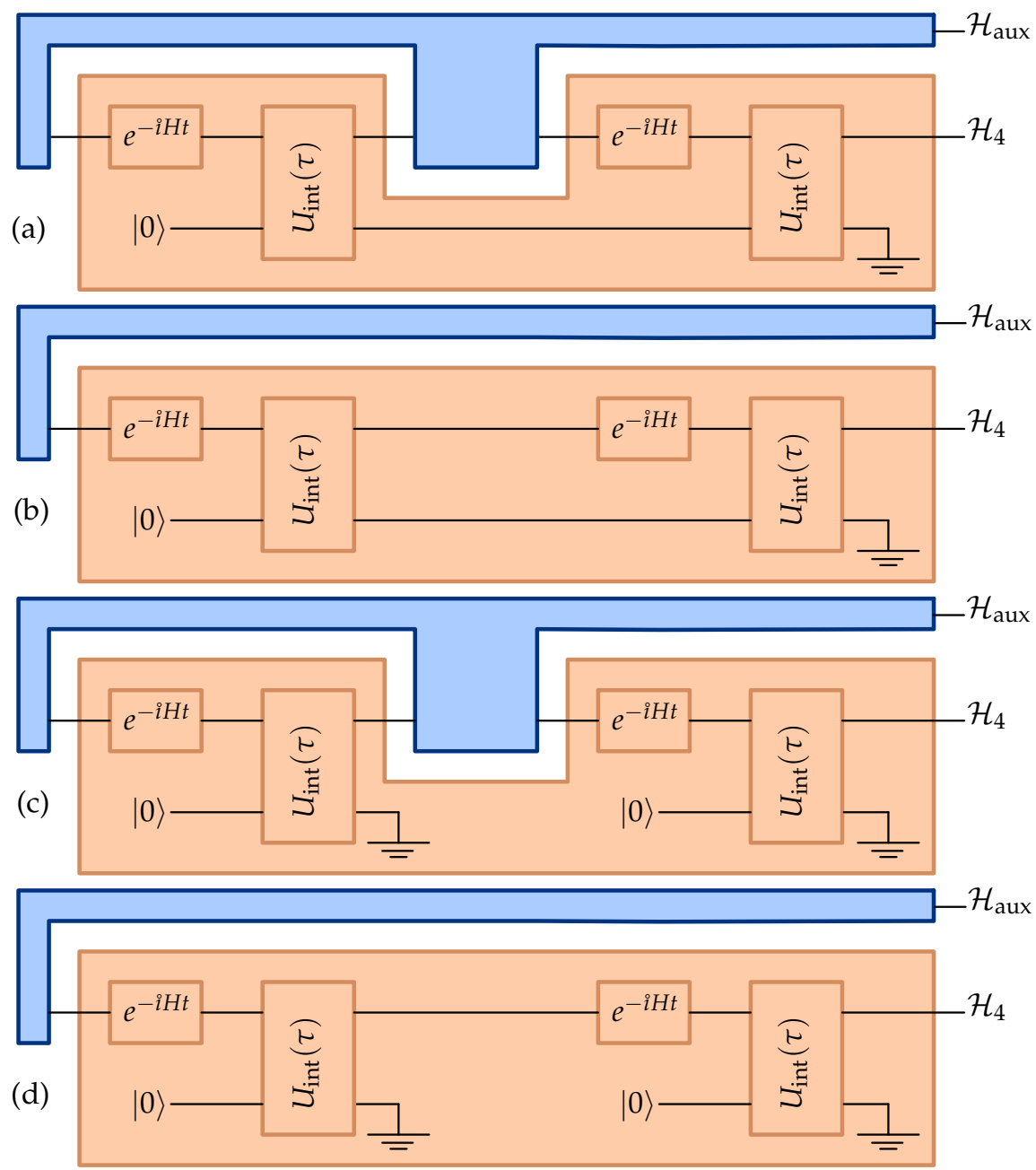

Figure 7. Collision model with $N=2$ interactions. We distinguish between non-Markovian processes ( $\mathrm{a}, \mathrm{b})$ and Markov processes (c, d). In addition we distinguish between operations with control $(a, c)$ and without control (b, d).

\section{Different interactions}

We give some examples [40] for unitary interactions $U=U_{\text {int }}(\tau)\left(\mathbb{1}_{E} \otimes e^{-i H t}\right)$.

\section{a. Partial SWAP}

Consider a qubit in $\mathcal{H}_{S}$ interacting with a partial swap with a two-dimensional environment $\mathcal{H}_{E}:$ In the basis $\left\{|00\rangle_{E S},|01\rangle_{E S},|10\rangle_{E S},|11\rangle_{E S}\right\}$ the swap is given as

$$
\text { SWAP }=\left(\begin{array}{llll}
1 & 0 & 0 & 0 \\
0 & 0 & 1 & 0 \\
0 & 1 & 0 & 0 \\
0 & 0 & 0 & 1
\end{array}\right) .
$$

If the system interacts with the environment with interaction strength $g$ and for time $\tau$ under this SWAP, the 
evolution is $U_{\operatorname{SWAP}}(\tau)=e^{-i g \tau \operatorname{SWAP}}$. We assume that the qubit's evolution is governed by a phase shift $H=\omega|1\rangle\langle 1|$, thus in the same basis as above, the time evolution of the joint system is

$$
U=\left(\begin{array}{cccc}
e^{-i g \tau} & 0 & 0 & 0 \\
0 & \cos (g \tau) e^{-i \omega t} & -i \sin (g \tau) & 0 \\
0 & -i \sin (g \tau) e^{-i \omega t} & \cos (g \tau) & 0 \\
0 & 0 & 0 & e^{-i(\omega t+g \tau)}
\end{array}\right)
$$

b. Partial CNOT with environment as control

We consider a partial CNOT as interaction. If we consider the environment as the control and the system as the target, the CNOT acts on the total system as

$$
\mathrm{CNOT}_{E}=\left(\begin{array}{llll}
1 & 0 & 0 & 0 \\
0 & 1 & 0 & 0 \\
0 & 0 & 0 & 1 \\
0 & 0 & 1 & 0
\end{array}\right)
$$

in the same basis as above. The corresponding unitary is $U_{\mathrm{CNOT}_{E}}(\tau)=e^{-i g \tau \mathrm{CNOT}_{E}}$ where $g$ is the interaction strength and $\tau$ the interaction time. We assume that the system undergoes a phase shift $H=\omega|1\rangle\langle 1|$ for time $t$ before the partial CNOT is applied. The resulting unitary is

$$
U_{\mathrm{CNOT}_{E}}(\tau)\left(\mathbb{1}_{E} \otimes e^{-i H t}\right)=\left(\begin{array}{cccc}
e^{-i g \tau} & 0 & 0 & 0 \\
0 & e^{-i(g \tau+\omega t)} & 0 & 0 \\
0 & 0 & \cos (g \tau) & -i \sin (g \tau) e^{-i \omega t} \\
0 & 0 & -i \sin (g \tau) & \cos (g \tau) e^{-i \omega t}
\end{array}\right) .
$$

If we initialise the environment in $|0\rangle_{E_{1}}$, we note that the partial CNOT does not have any effect. That is why we choose to initialise in $|+\rangle_{E_{1}}=\frac{1}{\sqrt{2}}\left(|0\rangle_{E_{1}}+|1\rangle_{E_{1}}\right)$. In order to be able to use the same setup as before, we set

$$
U=\left(U_{\mathrm{basis}} \otimes \mathbb{1}_{S}\right)^{\dagger} U_{\mathrm{CNOT}_{E}}(\tau)\left(\mathbb{1}_{E} \otimes e^{-i H t}\right)\left(U_{\mathrm{basis}} \otimes \mathbb{1}_{S}\right)
$$

with $U_{\text {basis }}$ given by

$$
U_{\mathrm{basis}}=\frac{1}{\sqrt{2}}\left(\begin{array}{cc}
1 & 1 \\
1 & -1
\end{array}\right)
$$

\section{c. Partial CNOT with system as control}

If we assume the system to control the CNOT and the environment to be the target, we start with

$$
\mathrm{CNOT}_{S}=\left(\begin{array}{llll}
1 & 0 & 0 & 0 \\
0 & 0 & 0 & 1 \\
0 & 0 & 1 & 0 \\
0 & 1 & 0 & 0
\end{array}\right),
$$


where we used the same basis as before. Analogously to the previous case, we then find $U=e^{-i g \tau \mathrm{CNOT}_{S}}\left(\mathbb{1}_{E} \otimes e_{S}^{-i H t}\right)$ to be

$$
U=\left(\begin{array}{cccc}
e^{-i g \tau} & 0 & 0 & 0 \\
0 & \cos (g \tau) e^{-i \omega t} & 0 & -i \sin (g \tau) e^{-i \omega t} \\
0 & 0 & e^{-i g \tau} & 0 \\
0 & -i \sin (g \tau) e^{-i \omega t} & 0 & \cos (g \tau) e^{-i \omega t}
\end{array}\right)
$$

\section{d. Bitflip channel}

Consider a system-environment interaction $H_{\mathrm{int}}=g X \otimes X$ with $g$ the interaction strength and $X$ the Pauli $X$ matrix. This interaction is a good approximation for situations in which the coupling to a single spin from the bath dominates over other interactions [41,42]. The environment starts in the completely mixed state $\rho_{E, 0}=\frac{1}{2} \mathbb{1}_{E}$, which corresponds to an equilibrium state at high temperature. Furthermore we assume that the system's initial state is uncorrelated with the environment: $\rho_{0}=\rho_{S, 0} \otimes \rho_{E, 0}$. Note that $e^{-i g \tau X \otimes X}=\cos (g \tau) \mathbb{1}_{S E}-i \sin (g \tau) X \otimes X$ and the unitary $U=U_{\text {int }}(\tau)\left(\mathbb{1}_{E} \otimes e^{-\imath H t}\right)$ is given by

$$
U=\left(\begin{array}{cccc}
\cos (g \tau) & 0 & 0 & -i \sin (g \tau) e^{-i \omega t} \\
0 & \cos (g \tau) e^{-i \omega t} & -i \sin (g \tau) & 0 \\
0 & -i \sin (g \tau) e^{-i \omega t} & \cos (g \tau) & 0 \\
-i \sin (g \tau) & 0 & 0 & \cos (g \tau) e^{-i \omega t}
\end{array}\right)
$$

We observe that the state after an interaction corresponds to

$$
\begin{aligned}
\rho_{S E}(\tau) & =\left(\cos ^{2}(g \tau) \rho_{S, 0}+\sin ^{2}(g \tau) X \rho_{S, 0} X\right) \otimes \frac{1}{2} \mathbb{1}_{E} \\
& +i \cos (g \tau) \sin (g \tau)\left(\rho_{S, 0} X+X \rho_{S, 0}\right) \otimes \frac{1}{2} X .
\end{aligned}
$$

The dynamics reduces on the system's space to

$$
\rho_{S}(\tau)=\operatorname{tr}_{E}\left[\rho_{S E}(\tau)\right]=\cos ^{2}(g \tau) \rho_{S, 0}+\sin ^{2}(g \tau) X \rho_{S, 0} X .
$$

Setting $p=\sin ^{2}(g \tau)$, we recover the bit flip channel

$$
\rho_{S}=(1-p) \rho_{S, 0}+p X \rho_{S, 0} X .
$$

\section{Results}

We implement the semidefinite program using CVX [43]. For the calculation, we choose $N=2,3,4$, the frequency $\omega=\frac{\pi}{10}$, the total time $t_{\text {tot }} \in[0,21]$ and the interaction strength $g=1$. This ensures that $\omega \cdot t_{\text {tot }}$ spans $[0,2 \pi]$. By repeating the simulation for different values of $\omega$, we see that the QFI and the optimal tester do not depend on $\omega$. This allows to probe the comb independently of the true value of $\omega$. We choose the sampling and interaction time of a single evolution to be $t=\tau=\frac{t_{\text {tot }}}{N}$. Some of the obtained plots are depicted in Figure 8 . The noiseless limit $J_{Q}\left(t_{\text {tot }}\right)=$ $t_{\text {tot }}^{2}$ is realised by setting the interaction strength $g=0$. We note that the non-Markovian case with control achieves the highest QFI for the partial SWAP. For the partial CNOT with the environment as control and the bitflip channel, it even achieves the noiseless scaling $J_{Q}=t_{\text {tot }}^{2}$. For $g \tau$ a multiple of $\pi$, the system-environment interaction becomes trivial and we recover the noiseless scaling for all scenarios. For $g \tau=\frac{2 k+1}{2} \pi$ with $k$ an integer, the interaction between system and environment is maximal; e.g., for the swap, all the information about $\theta$ is swapped to the environment and erased in the Markovian case.

Since the dual problem finds the optimal $h$ for $\Omega_{\theta}(h)$, we can insert it in the primal problem to find the optimal probe $T$. The optimal probes are diagonal. The data can be found on a repository [44]. Note that, e.g., for $N=2$ the probe is an element in $T \in \operatorname{Comb}\left[\left(\varnothing, \mathcal{H}_{1}\right),\left(\mathcal{H}_{2}, \mathcal{H}_{3}\right)\right]$, since the auxiliary space $\mathcal{H}_{\text {aux }}$ does not enter the SDP. In this work, we only exactly calculate the QFI of a generic quantum $N$-comb for $N \leq 4$, and calculation regarding the $N>5$ cases is likely to require new numerical methods and/or approximations. 
SWAP, $N=2$

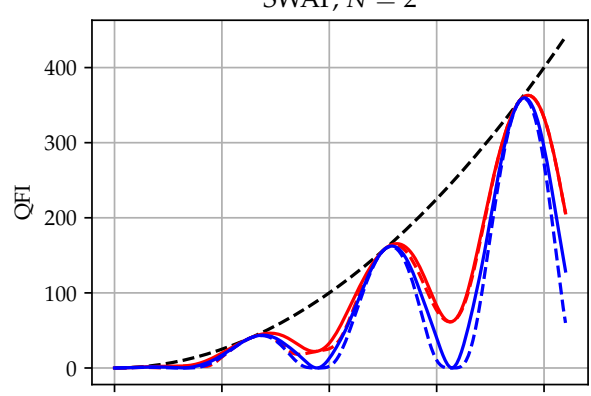

CNOT system, $N=2$
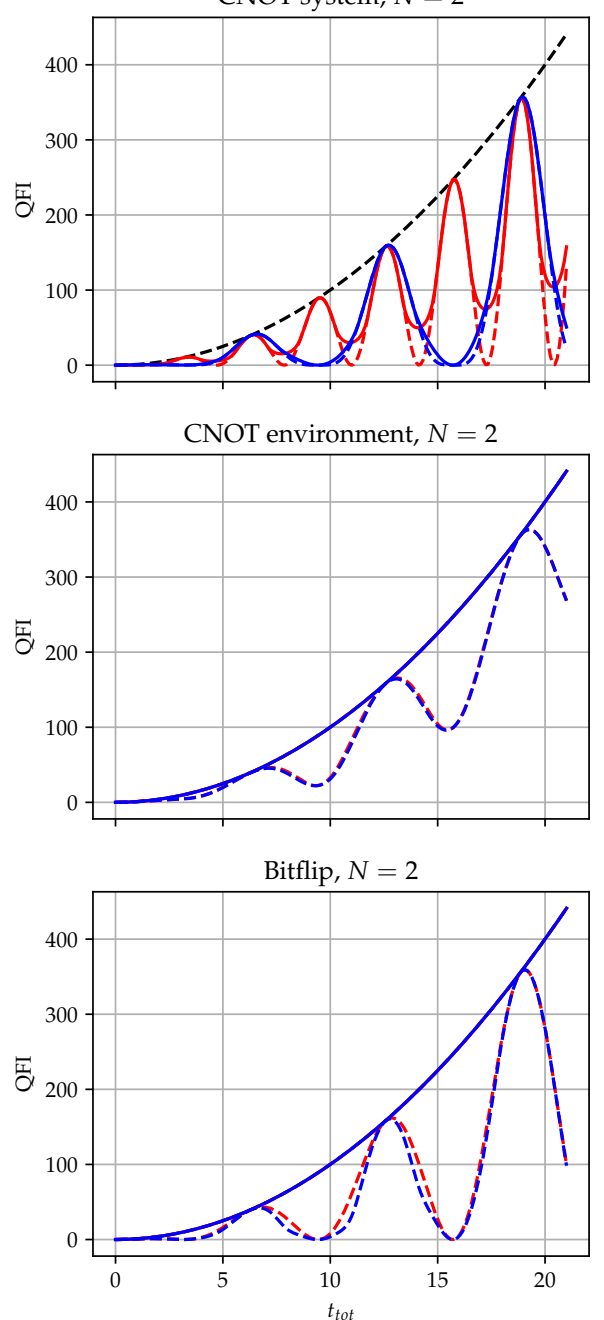

SWAP, $N=3$

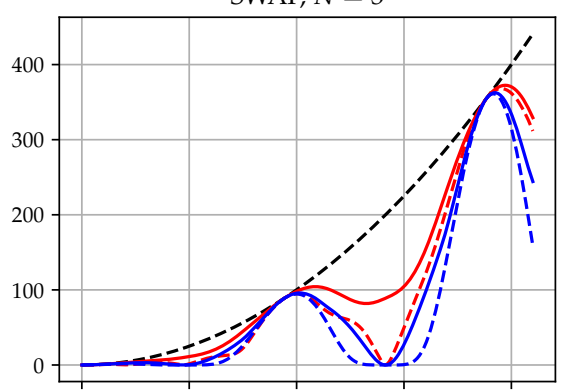

CNOT system, $N=3$

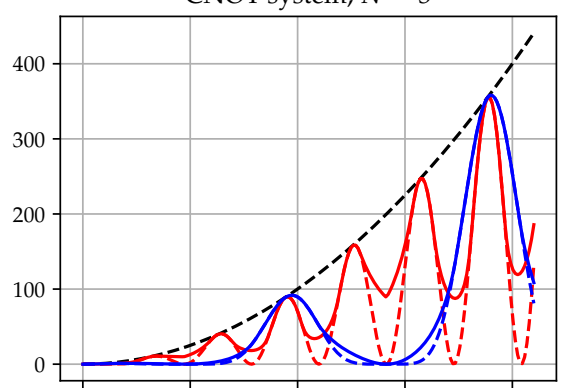

CNOT environment, $N=3$

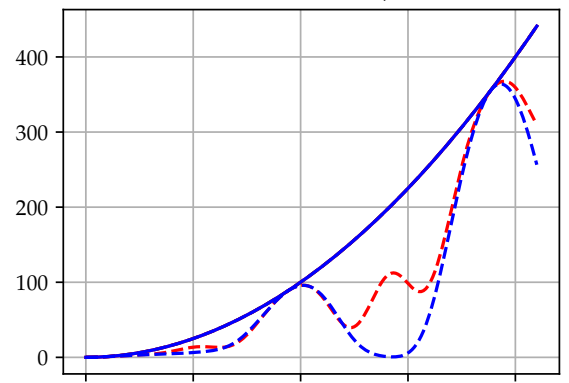

Bitflip, $N=3$

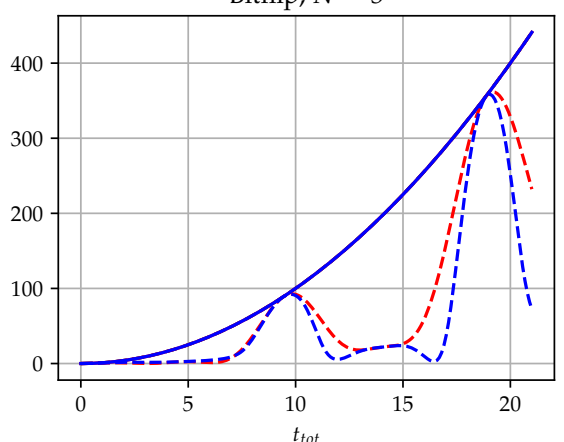

SWAP, $N=4$

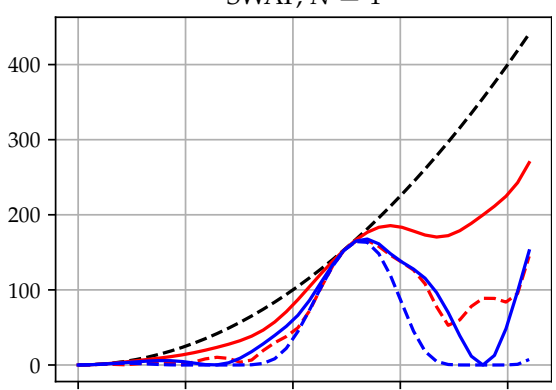

CNOT system, $N=4$

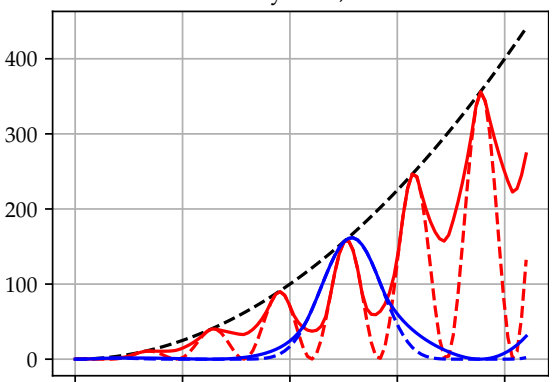

CNOT environment, $N=4$

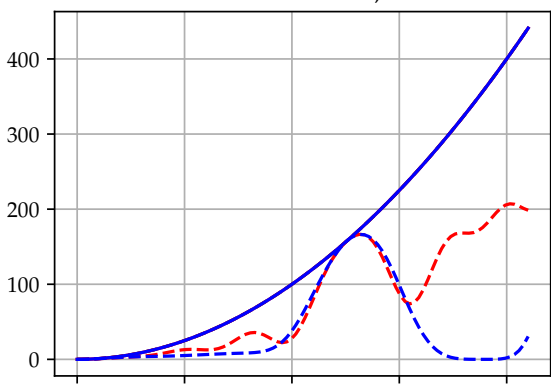

Bitflip, $N=4$

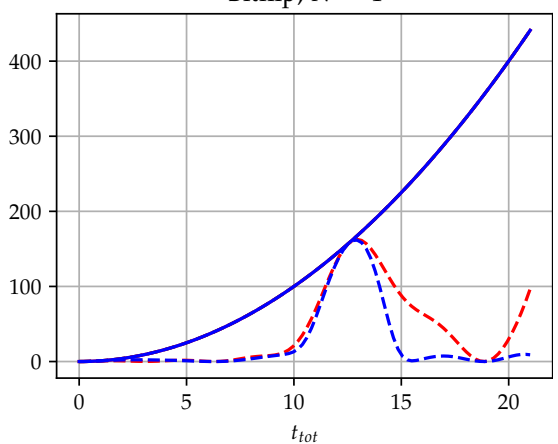

--- noiseless — non-Markov, control - - non-Markov, no control — Markov, control --- Markov, no control

Figure 8. Quantum Fisher information as a function of the total evolution time. The interaction time $\tau$ is chosen equal to the evolution time $t$. For the CNOT environment and the bitflip, the blue and red solid lines overlap with the black dashed line.

\section{Appendix C: QFI for adaptive channel estimation}

An interesting question in channel estimation arises by considering $N$ copies of a parametrised quantum channel $\mathcal{E}_{\theta}$. Here we show that the QFI corresponding to the optimal adaptive strategy for this task can be readily evaluated using our result.

The asymptotic limit of the QFI for $N \rightarrow \infty$ copies has been treated in Ref. [3, 30]. We can use our framework 
to model this question by considering the Choi operator $E_{\theta}$ of that quantum channel with decomposition $E_{\theta}=$ $\sum_{i}\left|E_{\theta, i}\right\rangle\left\langle E_{\theta, i}\right|$. We consider $N$ copies that act on subsequent Hilbert spaces $E_{\theta} \in \operatorname{Comb}\left[\left(\mathcal{H}_{2 k-1}, \mathcal{H}_{2 k}\right)\right]$ for $k=1, \ldots, N$. The resulting comb of these $N$ channels is the tensor product of the individual channels $C_{\theta}:=E_{\theta}^{\otimes N}$ and we find an ensemble decomposition $C_{\theta}=\sum_{\vec{i}}\left|C_{\theta, \vec{i}}\right\rangle\left\langle C_{\theta, \vec{i}}\right|$ (we use a vectorial index $\vec{i}=\left(i_{1}, \ldots, i_{N}\right)$ ) with

$$
\left|C_{\theta, \vec{i}=\left(i_{1}, \ldots, i_{N}\right)}\right\rangle=\bigotimes_{k=1}^{N}\left|E_{\theta, i_{k}}\right\rangle .
$$

We determine the performance operator of $C_{\theta}$ by computing the derivatives of $\left|C_{\theta, \vec{i}}\right\rangle$ :

$$
\left|\dot{C}_{\theta, \vec{i}}\right\rangle=\sum_{k=1}^{N}\left|E_{\theta, i_{1}}\right\rangle \ldots\left|E_{\theta, i_{k-1}}\right\rangle\left|\dot{E}_{\theta, i_{k}}\right\rangle\left|E_{\theta, i_{k+1}}\right\rangle \ldots\left|E_{\theta, i_{N}}\right\rangle
$$

We insert this derivative into

$$
\Omega_{\theta}^{N \text { copies }}:=4 \sum_{\vec{i}}\left|\dot{C}_{\theta, \vec{i}}\right\rangle\left\langle\dot{C}_{\theta, \vec{i}}\right|
$$

and note that $\Omega_{\theta}^{N \text { copies }}$ consists of the following terms

$$
\begin{aligned}
& \sum_{i_{k}}\left|\dot{E}_{\theta, i_{k}}\right\rangle\left\langle\dot{E}_{\theta, i_{k}}\right|=\frac{1}{4} \Omega_{\theta}, \\
& \sum_{i_{k}}\left|\dot{E}_{\theta, i_{k}}\right\rangle\left\langle E_{\theta, i_{k}}\right|=: \frac{1}{2}(\dot{E} E)_{\theta}, \\
& \sum_{i_{k}}\left|E_{\theta, i_{k}}\right\rangle\left\langle\dot{E}_{\theta, i_{k}}\right|=: \frac{1}{2}(E \dot{E})_{\theta}, \\
& \sum_{i_{k}}\left|E_{\theta, i_{k}}\right\rangle\left\langle E_{\theta, i_{k}}\right|=E_{\theta},
\end{aligned}
$$

where $\Omega_{\theta}$ is the performance operator of the channel $E_{\theta}$. More explicitly,

$$
\left.\Omega_{\theta}^{N \text { copies }}=\sum_{i+j+1=N} E_{\theta}^{\otimes i} \Omega_{\theta} E_{\theta}^{\otimes j}+\sum_{i+j+k+2=N} E_{\theta}^{\otimes i}\left[\left(\dot{E} E_{\theta}\right) E_{\theta}^{\otimes j}(E \dot{E})_{\theta}+(E \dot{E})_{\theta} E_{\theta}^{\otimes j}(\dot{E} E)_{\theta}\right)\right] E_{\theta}^{\otimes k} .
$$

Finally, according to Theorem 1 of the main text, the QFI of the optimal adaptive strategy is determined by $\Omega_{\theta}^{N \text { copies }}$ (or, more explicitly, the conditional comb min-entropy of $\Omega_{\theta}^{N \text { copies }}$ ). The optimal adaptive strategy of channel estimation can therefore be determined. 